\title{
ASYMPTOTIC BEHAVIOUR OF HOLOMORPHIC STRIPS
}

\author{
Joel W. ROBBIN ${ }^{\text {a }}$, Dietmar A. SALAMON ${ }^{\text {b }}$ \\ ${ }^{a}$ University of Wisconsin, Department of Methematics, Madison, WI 53 706, USA \\ ${ }^{\mathrm{b}}$ ETH-Zürich, Zürich, Switzerland
}

Received 10 May 2000

ABSTRACT. - The asymptotic behaviour of a finite energy pseudoholomorphic strip with Lagrangian boundary conditions in a symplectic manifold is determined by an eigenfunction of the linearized operator at the (transverse) intersection.

(C) 2001 L'Association Publications de l'Institut Henri Poincaré. Published by Elsevier B.V. All rights reserved

RÉSUMÉ. - Le comportement asymptotique d'une bande pseudoholomorphe d'énergie finie, à frontière dans une sous-variété Lagrangienne d'une variété symplectique, est déterminé par une fonction propre du problème linéarisé le long de l'intersection (transverse).

(C) 2001 L'Association Publications de l'Institut Henri Poincaré. Published by Elsevier B.V. All rights reserved

\section{Introduction}

This paper deals with the asymptotic behaviour of pseudoholomorphic strips in symplectic manifolds that satisfy Lagrangian boundary conditions. More precisely, let $(M, \omega)$ be a symplectic manifold and $L_{0}, L_{1} \subset M$ be closed (not necessarily compact) Lagrangian submanifolds that intersect transversally. Fix a $t$-dependent family of almost complex structures $J_{t}$ on $M$ that are compatible with $\omega$. We consider smooth maps $u: \mathbb{R}+i[0,1] \rightarrow M$ that satisfy the boundary value problem

$$
\partial_{s} u+J_{t}(u) \partial_{t} u=0, \quad u(\mathbb{R}) \subset L_{0}, \quad u(\mathbb{R}+i) \subset L_{1} .
$$

Such holomorphic strips were studied by Floer $[7,8]$ and he used them in his definition of the Floer homology of Lagrangian intersections. The standard theory of such holomorphic strips shows that if $u$ has finite energy then the limit

$$
p=\lim _{s \rightarrow \infty} u(s, t)
$$

exists and is an intersection point of $L_{0}$ and $L_{1}$.

E-mail address: robbin@math.wisc.edu (J.W. Robbin). 
Our main result (Theorem B) asserts that the limit

$$
v(t)=\lim _{s \rightarrow \infty} \frac{\partial_{s} u(s, t)}{\left\|\partial_{s} u\right\|} \in T_{p} M, \quad\left\|\partial_{s} u\right\|:=\sqrt{\int_{0}^{1}\left|\partial_{s} u(s, t)\right|^{2} d t},
$$

exists and, for some $\lambda>0$, satisfies the eigenvalue problem

$$
J_{t}(p) \partial_{t} v-\lambda v=0, \quad v(0) \in T_{p} L_{0}, \quad v(1) \in T_{p} L_{1} .
$$

It also asserts that there exists a constant $c>0$ such that

$$
\lim _{s \rightarrow \infty} \mathrm{e}^{\lambda s} \partial_{s} u(s, t)=c v(t)
$$

for every $t$. The convergence is exponential and in the $C^{\infty}$ topology.

There are four problems concerning the asymptotic behaviour of pseudoholomorphic curves. The first two refer to symplectic geometry and the last two to contact geometry.

(i) The asymptotic behaviour of a pseudoholomorphic cylinder twisted by a symplectomorphism that converges to a symplectic fixed point.

(ii) The asymptotic behaviour of a pseudoholomorphic strip with Lagrangian boundary conditions that converges to an intersection point.

(iii) The asymptotic behaviour of a pseudoholomorphic plane that converges to a closed characteristic, i.e. a closed integral curve of the Reeb vector field.

(iv) The asymptotic behaviour of a pseudoholomorphic half plane with Legendrian boundary conditions that converges to a characteristic chord, i.e. an integral curve of the Reeb vector field connecting the Legendrian submanifold to itself.

Problem (i) is relevant to the Floer homology of a symplectomorphism, (ii) is relevant to the Floer homology of a pair of Lagrangian submanifolds, (iii) is relevant to contact homology, and (iv) is relevant to the relative contact homology of a Legendrian submanifold. Our results are directed primarily at problem (ii). Problem (i) is a special case of (ii): use the diagonal and the graph of the symplectomorphism as the two Lagrangian submanifolds. However, although there is a strong similarity in the techniques used in the proofs, there seems to be no easy way to reduce any of the remaining problems to (ii) or vice versa.

There are two facets to each of the above problems, namely the existence of the limit and the asymptotic behaviour in terms of eigenvectors of the linearized operator. To establish the latter is considerably harder than the former. In (i) and (ii) the existence of the limit is an easy consequence of Gromov compactness. The convergence is exponential, see for example [17] for an exposition in the setting of problem (i). The existence of the limit in the setting of (iii) was established by Hofer [12] and used in his proof of the Weinstein conjecture in dimension three. The existence of the limit in the setting of (iv) was established in [6] and used in the definition of the Floer homology of a pair consisting of a Legendrian and a pre-Lagrangian submanifold.

The finer asymptotic behaviour in terms of the eigenvalues was treated by HoferWysocki-Zehnder [13] in the case of problem (iii) and by Abbas [1] in the case 
of problem (iv). Both papers only deal with three dimensional contact manifolds. In Appendix D.2 we explain in more detail the relation between our results and those of Abbas [1].

Our result is inspired by the work of Vin de Silva [18] on the Floer homology for Lagrangian intersections of two embedded loops in a Riemann surface. In this case De Silva gave a combinatorial description of the Floer homology in terms of embedded half discs (lunes). To prove that his combinatorial description agrees with Floer's definition of the Floer homology groups one has to establish a one-to-one correspondence between de Silva's lunes and Floer's holomorphic strips. The proof of this one-to-one correspondence seems to require Theorem $\mathrm{C}$ below, which establishes the asymptotic behaviour in dimension two. We emphasize that Theorem $\mathrm{C}$, and hence the one-to-one correspondence between lunes and holomorphic strips, is easy to prove whenever the two embedded loops agree with straight lines in some holomorphic coordinate chart near each intersection. However, the proof in the general case is considerably harder and apparently requires the analysis of the asymptotic behaviour carried out in this paper.

We view the combinatorial definition of the relative contact homology of a Legendrian knot (see Chekanov [3] and Eliashberg [4]) as a contact anlogue of de Silva's combinatorial definition of the Floer homology on a Riemann surface. See the as yet unpublished work of Eliashberg-Givental-Hofer [5] for the analytic definition. The proof that the combinatorial and analytic definitions agree again requires Theorem C.

The present paper is organized as follows. In Section 1 we discuss holomorphic strips and state the main results. Theorem A about exponential decay is well known. However, the proof uses similar techniques as that of Theorem B and we include an exposition for the sake of completeness. The proof of Theorem B is based on the technique developed by Agmon-Nirenberg [2] for abstract differential operators of the form

$$
D=\frac{\partial}{\partial s}+A(s)+B(s)
$$

where $A(s)$ is an unbounded self-adjoint operator on a Hilbert space and converges to $A_{\infty}$ as $s$ tends to $\infty$ and $B(s)$ is skew-adjoint and tends to zero as $s$ tends to $\infty$. Section 2 explains how the holomorphic strips fit into such a framework, Section 3 discusses the technique of Agmon and Nirenberg, and Section 4 gives the proofs of Theorems A and B.

To give a self contained exposition we have included several appendices. They deal with differential inequalities of the form

$$
\Delta w \geqslant-c w^{(n+2) / n}
$$

for the Laplace operator in dimension $n$ (Appendix A), with apriori estimates for pseudoholomorphic curves (Appendix B), with $L^{2}$ estimates for the Cauchy-Riemann operator (Appendix C), and with the construction of a convenient metric near a totally real submanifold (Appendix D). This metric and the results of Appendix A are needed in the proof of the apriori estimates in Appendix B. In turn, the apriori estimates are needed in the proof of Theorem A. The $L^{2}$ estimates in Appendix $\mathrm{C}$ are needed at various places in the proofs of Theorems A and B. Appendix E explains an attempt to reduce problem (iv) to our results. 


\section{Holomorphic strips}

Throughout, $M$ denotes a smooth manifold, $\left\{J_{t}\right\}_{0 \leqslant t \leqslant 1}$ a smooth family of almost complex structures on $M$, and $L_{0}$ and $L_{1}$ are closed (not necessarily compact) transverse submanifolds of $M$ such that $L_{0}$ is totally real for $J_{0}$ and $L_{1}$ is totally real for $J_{1}$. Whenever convenient, we write

$$
J=J(t, p)=J_{t}(p) .
$$

We shall assume throughout that the following holds at each intersection point $p \in$ $L_{0} \cap L_{1}$.

HYPOTHESIS H. - There is a nondegenerate skew form

$$
T_{p} M \times T_{p} M \rightarrow \mathbb{R}:(v, w) \mapsto \omega_{p}(v, w)
$$

that renders each of the subspaces $T_{p} L_{0}$ and $T_{p} L_{1}$ Lagrangian, i.e.

$$
\omega_{p}(v, w)=0 \quad \text { for }(v, w) \in T_{p} L_{t} \times T_{p} L_{t}, t=0,1,
$$

and such that the form

$$
T_{p} M \times T_{p} M \rightarrow \mathbb{R}:(v, w) \mapsto \omega_{p}\left(v, J_{t}(p) w\right)
$$

is symmetric positive definite for $0 \leqslant t \leqslant 1$.

For example, this is the case when $\omega_{p}$ is the value at $p$ of a symplectic form on $M, L_{0}$ amd $L_{1}$ are Lagrangian submanifolds, and each $J_{t}$ is compatible with $\omega$.

Consider the half strip

$$
S:=[0, \infty)+i[0,1]=\{s+i t \mid 0 \leqslant s<\infty, 0 \leqslant t \leqslant 1\}
$$

and let

$$
\partial_{0} S:=[0, \infty), \quad \partial_{1} S:=[0, \infty)+i .
$$

We consider smooth maps $u: S \rightarrow M$ which are holomorphic in the sense that they satisfy the Cauchy-Riemann equations

$$
\partial_{s} u+J_{t}(u) \partial_{t} u=0
$$

and satisfy the boundary conditions

$$
u\left(\partial_{0} S\right) \subset L_{0}, \quad u\left(\partial_{1} S\right) \subset L_{1} .
$$

Theorem A.-Assume $M$ is compact and $u$ satisfies (CR) and (BC). Then the following are equivalent.

(I) $u$ has finite energy:

$$
E(u):=\int_{S}\left|\partial_{s} u\right|^{2}<\infty .
$$


(II) The limits

$$
p:=\lim _{s \rightarrow \infty} u(s, t), \quad 0=\lim _{s \rightarrow \infty} \partial_{s} u(s, t)
$$

exist uniformly in $t$.

(III) $\partial_{s} u$ decays exponentially in the $C^{\infty}$ topology, i.e. there are positive constants $\varepsilon$ and $c_{1}, c_{2}, c_{3}, \ldots$ such that, for all $s$ and $k$,

$$
\left\|\partial_{s} u\right\|_{C^{k}([s, \infty) \times[0,1])} \leqslant c_{k} \mathrm{e}^{-\varepsilon s} .
$$

Each condition (I)-(III) is independent of the choice of the Riemannian metric used to express it. When (III) holds it follows from (CR) that $\partial_{t} u$ and in fact all derivatives of $u$ decay exponentially. In the setting of [17] the equation contains an additional Hamiltonian perturbation; in that case the convergence of the $t$-derivatives does not follow from (III). We introduce the abbreviation

$$
\mathcal{S}=\left\{u \in C^{\infty}(S, M) \mid u \text { satisfies }(\mathrm{CR}),(\mathrm{BC}),(\mathrm{I})-(\mathrm{III})\right\} .
$$

THEOREM B. - Assume $u \in \mathcal{S}$ is nonconstant. Then there exist an eigenvalue $\lambda>0$ and a nonzero eigenfunction $v:[0,1] \rightarrow T_{p} M$ such that

$$
J_{t}(p) \partial_{t} v-\lambda v=0, \quad v(0) \in T_{p} L_{0}, \quad v(1) \in T_{p} L_{1},
$$

and

$$
v(t)=\lim _{s \rightarrow \infty} \mathrm{e}^{\lambda s} \partial_{s} u(s, t) .
$$

The convergence is with all derivatives, uniform in $t$, and exponential. Thus there exist a smooth function $w:[0, \infty) \times[0,1] \rightarrow T_{p} M$ and positive constants $\delta$ and $c_{0}, c_{1}, c_{2}, \ldots$ such that, for every $s \geqslant 0$, every $t \in[0,1]$, and every integer $k \geqslant 0$,

$$
u(s, t)=\exp _{p}\left(-\frac{1}{\lambda} \mathrm{e}^{-\lambda s} v(t)+w(s, t)\right), \quad\|w\|_{C^{k}([s, \infty) \times[0,1])} \leqslant c_{k} \mathrm{e}^{-(\lambda+\delta) s} .
$$

In particular, there exist positive constants $s_{0}$ and $c$ such that, for every $s \geqslant s_{0}$ and every $t \in[0,1]$,

$$
\frac{1}{c} \mathrm{e}^{-\lambda s} \leqslant\left|\partial_{s} u(s, t)\right| \leqslant c \mathrm{e}^{-\lambda s} .
$$

Consider the special case where $M=\mathbb{C}, J_{t}=i$, and $p=0$. The tangent spaces $T_{p} L_{0}$ and $T_{p} L_{1}$ are real lines through the origin. Let the angle from $T_{p} L_{0}$ to $T_{p} L_{1}$ be $\nu_{0} \in(0, \pi)$. Then the eigenfunctions and eigenvalues of the linearized operator have the form

$$
v(t)=c_{0} \mathrm{e}^{i\left(v_{0}-k \pi\right) t}, \quad \lambda=k \pi-v_{0},
$$

where $c_{0} \in T_{p} L_{0} \subset \mathbb{C}, c_{0} \mathrm{e}^{i \nu_{0}} \in T_{p} L_{1}$, and $k \in \mathbb{Z}$. Every such $v$ is an eigenfunction, however, only positive integers $k$ can occur in a limiting eigenfunction. We reformulate Theorem B in this case. 
THEOREM C. - Let $u: S \rightarrow \mathbb{C}$ be a holomorphic map which satisfies $u\left(\partial_{0} S\right) \subset L_{0}$, $u\left(\partial_{1} S\right) \subset L_{1}$, and

$$
\lim _{s \rightarrow \infty} u(s, t)=\lim _{s \rightarrow \infty} \partial_{s} u(s, t)=0
$$

uniformly in $t$. Then there exist a unique nonzero complex number $c_{0}$, a unique positive real number $v$, and $a \delta>0$ such that

$$
u(s+i t)=c_{0} \mathrm{e}^{-v(s+i t)}+\mathrm{O}\left(\mathrm{e}^{-(v+\delta) s}\right) .
$$

These numbers satisfy $c_{0} \in T_{0} L_{0}, c_{0} \mathrm{e}^{i v_{0}} \in T_{0} L_{1}, 0<v_{0}<\pi$, and $v=k \pi-v_{0}$ for some positive integer $k$.

As a warmup we give a direct proof of this result in the (very special) case where $L_{0}=\mathbb{R}$ and $L_{1}=\mathrm{e}^{i \nu_{0}} \mathbb{R}$ are straight lines. The boundary conditions assert that the function

$$
v(s, t):=\mathrm{e}^{-v_{0}(s+i t)} u(s, t)
$$

extends to a holomorphic function from $\mathbb{C}^{+}:=[0, \infty)+i \mathbb{R} \rightarrow \mathbb{C}$, still denoted by $v$, such that

$$
v(z+2 i)=v(z), \quad v(\bar{z})=\bar{v}(z) .
$$

Since $v$ has period $2 i$ there exists a holomorphic function $w: D \backslash\{0\} \rightarrow \mathbb{C}$, where $D:=\{\zeta \in \mathbb{C}|| \zeta \mid \leqslant 1\}$, such that

$$
v(z)=w\left(\mathrm{e}^{-\pi z}\right)
$$

for $z \in \mathbb{C}^{+}$. Since $u$ is bounded it follows that

$$
|w(\zeta)| \leqslant C|\zeta|^{-\nu_{0} / \pi}
$$

for all $\zeta \in D \backslash\{0\}$ and some constant $C>0$. By the removable singularity theorem, $w$ extends to a holomorphic function on $D$. Hence there exist a unique nonzero complex number $c_{0}$ and a unique integer $k>0$ such that

$$
w(\zeta)=c_{0} \zeta^{k}+\mathrm{O}\left(|\zeta|^{k+1}\right)
$$

Hence $u(s, t)=\mathrm{e}^{\nu_{0}(s+i t)} w\left(\mathrm{e}^{-\pi(s+i t)}\right)$ satisfies (1) with these constants $c_{0}, \nu_{0}, k$, and with $\delta=\pi$.

The proof in the case of general transverse smooth curves $L_{0}$ and $L_{1}$ in $\mathbb{C}$ is considerably harder. It is marginally easier than the general case handled by Theorem $B$ because a Riemann surface is Kähler. In the Kähler case with $J$ independent of $t$ Appendix A can be simplified as indicated in Remark B.2.

\section{The linearized Cauchy-Riemann operator}

Because the strip $S$ carries a prefered vector field $\partial / \partial s$, the usual Cauchy-Riemann equations (see [14]) take the special form (CR). Thus the left hand side of (CR) can 
be viewed as a section of the pullback bundle $u^{*} T M \rightarrow S$. The Cauchy-Riemann operator (i.e. the map which sends $u$ to the left hand side of $(\mathrm{CR})$ ) is thus a section of the vector bundle $C^{\infty}(S, T M) \rightarrow C^{\infty}(S, M)$; the solutions of $(\mathrm{CR})$ are the zeros of this section. Denote

$$
\mathcal{B}:=\left\{u \in C^{\infty}(S, M) \mid u \text { satisfies }(B C)\right\}
$$

and

$$
\mathcal{E}:=\left\{\xi \in C^{\infty}(S, T M) \mid \pi \circ \xi \in \mathcal{B}\right\},
$$

where $\pi: T M \rightarrow M$ is the projection. Thus $\mathcal{E}$ is a vector bundle over $\mathcal{B}$ with fibre ${ }^{1}$ $\mathcal{E}_{u}:=C^{\infty}\left(u^{*} T M\right)$. The tangent space $T_{u} \mathcal{B}$ is the set of vector fields $\xi \in C^{\infty}\left(u^{*} T M\right)$ that satisfy

$$
\xi(s, 0) \in T_{u(s, 0)} L_{0}, \quad \xi(s, 1) \in T_{u(s, 1)} L_{1} .
$$

It is a general principle that the derivative of a section of a vector bundle at a zero $u$ of that section gives a well defined linear map from the tangent space to the base at $u$ to the fiber over $u$. In the case at hand this derivative is the linearized Cauchy-Riemann operator $D_{u}: T_{u} \mathcal{B} \rightarrow \mathcal{E}_{u}$, given by

$$
D_{u} \xi=\nabla_{s} \xi+\left(\nabla_{\xi} J_{t}(u)\right) \partial_{t} u+J_{t}(u) \nabla_{t} \xi .
$$

Because $u$ satisfies (CR), this operator is independent of the choice of the connection. Because $u$ satisfies (BC) we have $\partial_{s} u \in T_{u} \mathcal{B}$ and $D_{u} \partial_{s} u=0$. In the following lemma the reader is cautioned to distinquish between the value $\left.J\right|_{t=0}$ of $J$ when $t=0$ and the standard complex structure $J_{0}$ on $\mathbb{R}^{2 n}=\mathbb{C}^{n}$. We denote by $\omega_{0}$ the standard symplectic form on $\mathbb{R}^{2 n}$.

LEMMA 2.1. - There is a neighborhood $U$ of $p$ in $M$ and a local trivialization

$$
[0,1] \times U \times \mathbb{R}^{2 n} \rightarrow T M:(t, q, v) \mapsto \Phi_{t}(q) v \in T_{q} M
$$

such that for $(t, q) \in[0,1] \times U$ we have

(i) $J_{t}(q) \Phi_{t}(q)=\Phi_{t}(q) J_{0}$;

(ii) $\Phi_{t}(q)\left(\mathbb{R}^{n} \times\{0\}\right)=T_{q} L_{t}$ for $t=0,1$;

(iii) $\omega_{p}\left(\Phi_{t}(p) v, \Phi_{t}(p) w\right)=\omega_{0}(v, w)$ for $v, w \in \mathbb{R}^{2 n}$.

Proof. - Choose a smooth path $\left\{\Lambda_{t}\right\}_{0 \leqslant t \leqslant 1}$ of Lagrangian subspaces of $\left(T_{p} M, \omega_{p}\right)$ such that

$$
\Lambda_{0}=T_{p} L_{0}, \quad \Lambda_{1}=T_{p} L_{1} .
$$

Now choose smooth functions $e_{i}:[0,1] \rightarrow T_{p} M, i=1, \ldots, n$, such that, for every $t \in[0,1]$, the vectors $e_{1}(t), \ldots, e_{n}(t)$ form an orthonormal basis of $\Lambda_{t}$ with respect to the inner product $\omega_{p}\left(\cdot, J_{t}(p) \cdot\right)$. Define

$$
e_{i+n}(t):=J_{t}(p) e_{i}(t)
$$

\footnotetext{
${ }^{1}$ For a vector bundle $E \rightarrow B$ we denote by $C^{\infty}(E)$ the space of sections.
} 
for $i=1, \ldots, n$ and $t \in[0,1]$. Then the linear map $\Phi(t): \mathbb{R}^{2 n} \rightarrow T_{p} M$ defined by

$$
\Phi(t) v:=\sum_{i=1}^{2 n} v_{i} e_{i}(t)
$$

for $v=\left(v_{1}, \ldots, v_{2 n}\right) \in \mathbb{R}^{2 n}$ identifies $\mathbb{R}^{n} \times\{0\}$ with $\Lambda_{t}, J_{0}$ with $J_{t}(p)$, and $\omega_{0}$ with $\omega_{p}$. Now choose trivializations of $T L_{0}$ and $T L_{1}$ near $p$ that agree with the given isomorphisms $\mathbb{R}^{n} \rightarrow \Lambda_{t}$ for $t=0$ and $t=1$, respectively. Next extend these to trivializations of $T_{L_{0}} M$ and $T_{L_{1}} M$ that identify $J_{0}$ and $J_{1}$, respectively, with the standard complex structure on $\mathbb{R}^{2 n}$ and agree with $\Phi(t)$ for $t=0$, 1 . Finally extend the trivializations of $T_{L_{0}} M$ and $T_{L_{1}} M$ to a smooth family $\left\{\Phi_{t}\right\}_{0 \leqslant t \leqslant 1}$ of trivialization of $T M$ over a neighbourhood of $p$ such that $\Phi_{t}$ identifies $J_{t}$ with the standard complex structure on $\mathbb{R}^{2 n}$ and agrees with $\Phi(t)$ at $p$.

Assume part II of Theorem A and let $U \subset M$ be as in Lemma 2.1. Fix $u \in \mathcal{S}$ and assume without loss of generality that $u(s, t) \in U$ for all $s$ and $t$. Define the function $S:[0, \infty) \times[0,1] \rightarrow \mathbb{R}^{2 n \times 2 n}$ by the condition that

$$
\Phi_{t}(u)\left(\partial_{s} \xi+J_{0} \partial_{t} \xi+S \xi\right)=D_{u}\left(\Phi_{t}(u) \xi\right)
$$

holds for every smooth function $\xi:[0, \infty) \times[0,1] \rightarrow \mathbb{R}^{2 n}$. Here the right hand side of (4) is defined by substituting $\Phi_{t}(u) \xi$ for $\xi$ in (3). Define $S_{\infty}:[0,1] \rightarrow \mathbb{R}^{2 n \times 2 n}$ by

$$
\Phi_{t}(p) S_{\infty}(t):=J_{t}(p) \partial_{t} \Phi_{t}(p) .
$$

LEMmA 2.2. - In this notation the following holds. The matrix $S_{\infty}(t)$ is symmetric for every $t$ and there exists a constant $c>0$ such that

$$
\left\|S(s, t)-S_{\infty}(t)\right\| \leqslant c\left(\left|\partial_{s} u(s, t)\right|+d(u(s, t), p)\right)
$$

for every $s \geqslant 0$ and every $t \in[0,1]$. Moreover, if u satisfies a uniform $C^{k}$-bound for some integer $k \geqslant 1$, then there exists a constant $c_{k}>0$ such that

$$
\left\|S-S_{\infty}\right\|_{C^{k}([s, \infty) \times[0,1])} \leqslant c\left(\left\|\partial_{s} u\right\|_{C^{k}([s, \infty) \times[0,1])}+\sup _{s^{\prime} \geqslant s, 0 \leqslant t \leqslant 1} d\left(u\left(s^{\prime}, t\right), p\right)\right)
$$

for every $s \geqslant 0$.

Proof. - By (i) and (iii) in Lemma 2.1, we have

$$
\begin{aligned}
\omega_{0}\left(v, J_{0} S_{\infty}(t) w\right) & =\omega_{p}\left(\Phi_{t}(p) v, \Phi_{t}(p) J_{0} S_{\infty}(t) w\right) \\
& =\omega_{p}\left(\Phi_{t}(p) v, J_{t}(p) \Phi_{t}(p) S_{\infty}(t) w\right) \\
& =-\omega_{p}\left(\Phi_{t}(p) v, \partial_{t} \Phi_{t}(p) w\right) \\
& =\omega_{p}\left(\partial_{t} \Phi_{t}(p) v, \Phi_{t}(p) w\right) \\
& =-\omega_{p}\left(J_{t}(p) \Phi_{t}(p) S_{\infty}(t) v, \Phi_{t}(p) w\right) \\
& =\omega_{p}\left(\Phi_{t}(p) S_{\infty}(t) v, J_{t}(p) \Phi_{t}(p) w\right)
\end{aligned}
$$




$$
\begin{aligned}
& =\omega_{p}\left(\Phi_{t}(p) S_{\infty}(t) v, \Phi_{t}(p) J_{0} w\right) \\
& =\omega_{0}\left(S_{\infty}(t) v, J_{0} w\right)
\end{aligned}
$$

for $v, w \in \mathbb{R}^{2 n}$. Hence $S_{\infty}(t)$ is a symmetric matrix for every $t$. Inequality (6) follows from the identity

$$
\Phi_{t}(u) S v=\nabla_{S}\left(\Phi_{t}(u) v\right)+J_{t}(u) \nabla_{t}\left(\Phi_{t}(u) v\right)+\left(\nabla_{\Phi_{t}(u) v} J_{t}(u)\right) \partial_{t} u .
$$

For $k \geqslant 1$ estimate (7) follows by differentiating this identity. In particular,

$$
\begin{aligned}
\Phi_{t}(u)\left(\partial_{s} S\right) v= & \nabla_{s}\left(\Phi_{t}(u) S v\right)-\left(\nabla_{s} \Phi_{t}(u)\right) S v \\
= & \nabla_{s} \nabla_{s}\left(\Phi_{t}(u) v\right)+\nabla_{s}\left(J_{t}(u) \nabla_{t}\left(\Phi_{t}(u) v\right)\right) \\
& +\nabla_{s}\left(\left(\nabla_{\Phi_{t}(u) v} J_{t}(u)\right) \partial_{t} u\right)-\left(\nabla_{s} \Phi_{t}(u)\right) S v .
\end{aligned}
$$

Since $\nabla_{s} \partial_{t} u=\nabla_{s}\left(J \partial_{s} u\right)=\left(\nabla_{s} J\right) \partial_{s} u+J \nabla_{s} \partial_{s} u$, this implies (7) for $k=1$.

Consider the Hilbert spaces

$$
H:=L^{2}\left([0,1], \mathbb{R}^{2 n}\right)
$$

and

$$
V:=\left\{\xi \in W^{1,2}\left([0,1], \mathbb{R}^{2 n}\right) \mid \xi(0), \xi(1) \in \mathbb{R}^{n} \times\{0\}\right\} .
$$

Let $A(s): V \rightarrow H$ and $A_{\infty}: V \rightarrow H$ be defined by

$$
A(s):=J_{0} \partial_{t}+\frac{1}{2}\left(S(s, t)+S(s, t)^{T}\right), \quad A_{\infty}:=J_{0} \partial_{t}+S_{\infty},
$$

These operators are self-adjoint, as unbounded linear operators on $H$. Define $B(s): H \rightarrow$ $H$ by

$$
B(s):=\frac{1}{2}\left(S(s, t)-S(s, t)^{T}\right) .
$$

LEMma 2.3. - Continue to assume (II) in Theorem A and use the notation introduced in Eqs. (4), (5), (8), and (9). Then $A(s)-A_{\infty}, \dot{A}(s)$ and $B(s)$ are bounded linear operators on $H, A_{\infty}: V \rightarrow H$ is bijective, and there exists a constant $c>0$ such that, for every $s \geqslant 0$,

$$
\begin{gathered}
\left\|A(s)-A_{\infty}\right\|_{\mathcal{L}(H)}+\|B(s)\|_{\mathcal{L}(H)} \leqslant c \sup _{0 \leqslant t \leqslant 1}\left(\left|\partial_{s} u(s, t)\right|+d(u(s, t), p)\right), \\
\|\dot{A}(s)\|_{\mathcal{L}(H)} \leqslant c \sup _{0 \leqslant t \leqslant 1}\left(\left|\nabla_{s} \partial_{s} u(s, t)\right|+\left|\partial_{s} u(s, t)\right|+d(u(s, t), p)\right) .
\end{gathered}
$$

Proof. - Inequalities (10) and (11) follow immediately from Lemma 2.2. We prove that $A_{\infty}$ is injective. Let $\xi \in V$ such that $A_{\infty} \xi=0$. Then the function $[0,1] \rightarrow$ $T_{p} M: t \mapsto \Phi_{t}(p) \xi(t)$ satisfies

$$
\Phi_{0}(p) \xi(0) \in T_{p} L_{0}, \quad \Phi_{1}(p) \xi(1) \in T_{p} L_{1}
$$


and

$$
\begin{aligned}
\partial_{t}\left(\Phi_{t}(p) \xi(t)\right) & =\Phi_{t}(p) \partial_{t} \xi(t)-J_{t}(p) \Phi_{t}(p) S_{\infty}(t) \xi(t) \\
& =\Phi_{t}(p)\left(\partial_{t} \xi(t)-J_{0} S_{\infty}(t) \xi(t)\right) \\
& =0 .
\end{aligned}
$$

Since $L_{0}$ and $L_{1}$ intersect transversally at $p$ it follows that $\xi=0$ as claimed.

We prove that $A_{\infty}$ is surjective. Let $\Psi_{\infty}:[0,1] \times[0,1] \rightarrow \mathrm{Sp}(2 n)$ be the fundamental solution of the operator $J_{0} \partial_{t}+S_{\infty}$, i.e.

$$
J_{0} \partial_{t} \Psi_{\infty}\left(t, t^{\prime}\right)+S_{\infty}(t) \Psi_{\infty}\left(t, t^{\prime}\right)=0, \quad \Psi_{\infty}(t, t)=\mathbb{1} .
$$

Denote $\Lambda_{0}:=\mathbb{R}^{n} \times\{0\}$. Since $A_{\infty}$ is injective the map

$$
\Lambda_{0} \times \Lambda_{0} \rightarrow \mathbb{R}^{2 n}:\left(\xi_{0}, \xi_{1}\right) \mapsto \xi_{1}-\Psi_{\infty}(1,0) \xi_{0}
$$

is bijective. Given $\eta \in H$ define $\left(\xi_{0}, \xi_{1}\right) \in \Lambda_{0} \times \Lambda_{0}$ by

$$
\xi_{1}-\Psi_{\infty}(1,0) \xi_{0}:=-\int_{0}^{1} J_{0} \Psi_{\infty}\left(1, t^{\prime}\right) \eta\left(t^{\prime}\right) d t^{\prime} .
$$

Define $\xi:[0,1] \rightarrow \mathbb{R}^{2 n}$ by

$$
\xi(t):=\Psi_{\infty}(t, 0) \xi_{0}-\int_{0}^{t} J_{0} \Psi_{\infty}\left(t, t^{\prime}\right) \eta\left(t^{\prime}\right) d t^{\prime} .
$$

Then $\xi \in V$ and $A_{\infty} \xi=\eta$.

\section{Operators on Hilbert spaces}

Let $V$ and $H$ be separable Hilbert spaces such that

$$
V \subset H .
$$

Suppose that $V$ is a dense subset of $H$ and that the inclusion $V \hookrightarrow H$ is a compact linear operator. Assume without loss of generality that

$$
\|\xi\|_{H} \leqslant\|\xi\|_{V}
$$

for every $\xi \in V$. Throughout we denote by $\langle\cdot, \cdot\rangle$ the inner product on $H$ and by

$$
\|\cdot\|=\|\cdot\|_{H}
$$

the norm on $H$. (We never use the inner product or the norm on $V$.) Let $\mathcal{L}(V, H)$ denote the space of bounded linear operators from $V$ to $H$ and $\mathcal{L}(H)$ denote the space of 
bounded linear operators from $H$ to itself. Throughout this section let $A_{\infty} \in \mathcal{L}(V, H)$, let $[0, \infty) \rightarrow \mathcal{L}(V, H): s \mapsto A(s)$ be a continuously differentiable function, and let $[0, \infty) \rightarrow \mathcal{L}(H): s \mapsto B(s)$ be a continuous function. We shall denote by $\dot{A}(s)$ the derivative of $A(s)$ with respect to $s$. We impose the following conditions.

(a) $A(s)$ is symmetric for every $s$, i.e.

$$
\langle A(s) \xi, \eta\rangle=\langle\xi, A(s) \eta\rangle
$$

for $\xi, \eta \in V$. Moreover, the operators $A(s)-A_{\infty}$ and $\dot{A}(s)$ extend to bounded linear operators on $H, A_{\infty}: V \rightarrow H$ is bijective, and

$$
\lim _{s \rightarrow \infty}\left\|A(s)-A_{\infty}\right\|=\lim _{s \rightarrow \infty}\|\dot{A}(s)\|=0,
$$

where $\|\cdot\|$ denotes the operator norm on $\mathcal{L}(H)$.

(b) $B(s)$ is skew-symmetric for every $s$, i.e.

$$
\langle\xi, B(s) \eta\rangle=-\langle B(s) \xi, \eta\rangle
$$

for $\xi, \eta \in H$, and

$$
\lim _{s \rightarrow \infty}\|B(s)\|=0 .
$$

Lemma 3.1. - Assume (a) and (b) and let $\xi:[0, \infty) \rightarrow H$ and $\eta:[0, \infty) \rightarrow H$ be continuously differentiable functions. Suppose $\xi(s) \in V$ and

$$
\dot{\xi}(s)+A(s) \xi(s)+B(s) \xi(s)=\eta(s)
$$

for every $s \geqslant 0$. Suppose further that there exist positive constants $C$ and $\varepsilon$ such that

$$
\|\eta(s)\|+\|\dot{\eta}(s)\| \leqslant C \mathrm{e}^{-\varepsilon s}
$$

for every $s \geqslant 0$. Then there exist positive constants $c$ and $\delta$ such that

$$
\|\xi(s)\| \leqslant c \mathrm{e}^{-\delta s}
$$

for every $s \geqslant 0$.

Proof. - We suppress the argument $s$ whenever convenient. Consider the function

$$
\alpha(s):=\frac{1}{2}\|\xi(s)\|^{2} .
$$

Since $B(s)$ is skew-symmetric it follows that

$$
\dot{\alpha}=\langle\xi, \dot{\xi}\rangle=\langle\xi, \eta-A \xi\rangle,
$$

and hence 


$$
\begin{aligned}
\ddot{\alpha} & =\langle\dot{\xi}, \eta-2 A \xi\rangle+\langle\xi, \dot{\eta}-\dot{A} \xi\rangle \\
& =2\|A \xi\|^{2}+\|\eta\|^{2}-\langle A \xi, 3 \eta\rangle-\langle B \xi, \eta\rangle+\langle 2 B \xi, A \xi\rangle+\langle\xi, \dot{\eta}-\dot{A} \xi\rangle \\
& \geqslant\|A \xi\|^{2}-\frac{5}{2}\|B \xi\|^{2}-4\|\eta\|^{2}+\langle\xi, \dot{\eta}-\dot{A} \xi\rangle \\
& \geqslant\|A \xi\|^{2}-\left(\frac{5}{2}\|B\|^{2}+\|\dot{A}\|\right)\|\xi\|^{2}-4\|\eta\|^{2}+\langle\xi, \dot{\eta}\rangle .
\end{aligned}
$$

Here $\|B\|$ and $\|\dot{A}\|$ are understood as the operator norms on $\mathcal{L}(H)$. By (a), there exists a constant $\delta \in(0, \varepsilon)$ such that $\delta \leqslant 1 / 2$ and

$$
\left\|A_{\infty} v\right\| \geqslant 3 \delta\|v\|
$$

for every $v \in V$. By (a) and (b), there exists a constant $s_{0}>0$ such that

$$
\frac{5}{2}\|B(s)\|^{2}+\|\dot{A}(s)\| \leqslant \delta^{2}, \quad\left\|A(s)-A_{\infty}\right\| \leqslant \delta
$$

for every $s \geqslant s_{0}$. Hence

$$
\|A(s) v\| \geqslant\left\|A_{\infty} v\right\|-\left\|\left(A(s)-A_{\infty}\right) v\right\| \geqslant 2 \delta\|v\|
$$

for $s \geqslant s_{0}$ and $v \in V$. Hence, by (14) and (13), we have

$$
\begin{aligned}
\ddot{\alpha}(s) & \geqslant\|A(s) \xi(s)\|^{2}-\delta^{2}\|\xi(s)\|^{2}-4\|\eta(s)\|^{2}-\langle\xi(s), \dot{\eta}(s)\rangle \\
& \geqslant 3 \delta^{2}\|\xi(s)\|^{2}-4\|\eta(s)\|^{2}-\langle\xi(s), \dot{\eta}(s)\rangle \\
& \geqslant 2 \delta^{2}\|\xi(s)\|^{2}-4\|\eta(s)\|^{2}-\frac{1}{\delta^{2}}\|\dot{\eta}(s)\|^{2} \\
& \geqslant 2 \delta^{2}\|\xi(s)\|^{2}-\frac{1}{\delta^{2}}\left(\|\eta(s)\|^{2}+\|\dot{\eta}(s)\|^{2}\right) \\
& \geqslant 2 \delta^{2}\|\xi(s)\|^{2}-\frac{C^{2}}{\delta^{2}} \mathrm{e}^{-2 \varepsilon s} \\
& =(2 \delta)^{2} \alpha(s)-c_{0} \mathrm{e}^{-2 \varepsilon s}
\end{aligned}
$$

for $s \geqslant s_{0}$, where $c_{0}:=C^{2} / \delta^{2}$. Consider the function

$$
\beta(s):=\alpha(s)+\frac{c_{0} \mathrm{e}^{-2 \varepsilon s}}{(2 \varepsilon)^{2}-(2 \delta)^{2}} .
$$

This function satisfies

$$
\begin{aligned}
\ddot{\beta}(s) & =\ddot{\alpha}(s)+\frac{c_{0}(2 \varepsilon)^{2} \mathrm{e}^{-2 \varepsilon s}}{(2 \varepsilon)^{2}-(2 \delta)^{2}} \\
& \geqslant(2 \delta)^{2} \alpha(s)+\frac{c_{0}(2 \varepsilon)^{2} \mathrm{e}^{-2 \varepsilon s}}{(2 \varepsilon)^{2}-(2 \delta)^{2}}-c_{0} \mathrm{e}^{-2 \varepsilon s} \\
& =(2 \delta)^{2} \beta(s)
\end{aligned}
$$


for $s \geqslant s_{0}$. We prove that

$$
\dot{\beta}(s)+2 \delta \beta(s) \leqslant 0
$$

for every $s \geqslant s_{0}$. Suppose, otherwise, that $\dot{\beta}\left(s_{1}\right)+2 \delta \beta\left(s_{1}\right)>0$ for some $s_{1} \geqslant s_{0}$. Then, by (15),

$$
\dot{\beta}(s)+2 \delta \beta(s) \geqslant \mathrm{e}^{2 \delta s} c_{1}
$$

for every $s \geqslant s_{1}$ and some positive constant $c_{1}$. This implies

$$
\frac{d}{d s} \mathrm{e}^{2 \delta s} \beta(s) \geqslant \mathrm{e}^{4 \delta s} c_{1}
$$

for $s \geqslant s_{1}$. Integrating this inequality gives $\beta(s) \geqslant \mathrm{e}^{2 \delta s} c_{2}-c_{3}$ for every $s \geqslant s_{1}$ and some positive constants $c_{2}$ and $c_{3}$. This contradicts our assumption that $\|\xi(s)\|$ does not diverge to $\infty$ as $s$ tends to infinity. Thus we have proved (16). Write this inequality in the form

$$
\frac{d}{d s} \mathrm{e}^{2 \delta s} \beta(s) \leqslant 0 .
$$

With $c_{4}:=\mathrm{e}^{\delta s_{0}} \sqrt{2 \beta\left(s_{0}\right)}$ it follows that

$$
\|\xi(s)\| \leqslant \sqrt{2 \alpha(s)} \leqslant \sqrt{2 \beta(s)} \leqslant c_{4} \mathrm{e}^{-\delta s}
$$

for $s \geqslant s_{0}$. This proves the lemma.

Remark 3.2. - Assume the situation of Lemma 3.1 with $\eta=0$ and $C=0$. Then inequality (16) has the form

$$
\langle\xi(s), A(s) \xi(s)\rangle \geqslant \delta\|\xi(s)\|^{2}
$$

for $s \geqslant s_{0}$.

The next lemma is a simplified form of a theorem by Agmon and Nirenberg [2]. They used this technique to establish unique continuation for solutions of elliptic and parabolic partial differential equations.

Lemma 3.3 (Agmon-Nirenberg). - Assume that A, B, and $\xi$ satisfy the hypotheses of Lemma 3.1 with $\eta=0$ and that $\xi$ is nonconstant. Then $\xi(s) \neq 0$ for every $s \geqslant 0$ and the functions

$$
v(s):=\frac{\xi(s)}{\|\xi(s)\|}, \quad \lambda(s):=\langle v(s), A(s) v(s)\rangle
$$

satisfy

$$
\dot{\lambda}(s) \leqslant\|B(s)\|^{2}+\|\dot{A}(s)\|-\|A(s) v(s)-\lambda(s) v(s)\|^{2} .
$$

Moreover, if

$$
N:=\lambda(0)+\int_{0}^{\infty}\left(\|B(s)\|^{2}+\|\dot{A}(s)\|\right) d s<\infty
$$


then

$$
\|\xi(s)\| \geqslant \mathrm{e}^{-N s}\|\xi(0)\|
$$

for every $s \geqslant 0$.

Proof. - The formula (18) defines functions $v: \Omega \rightarrow V$ and $\lambda: \Omega \rightarrow \mathbb{R}$, where

$$
\Omega:=\{s \geqslant 0 \mid \xi(s) \neq 0\} .
$$

In the following we suppress the argument $s$. The derivative of $v$ is given by

$$
\dot{v}=(\lambda-A) v-B v
$$

Hence

$$
\begin{aligned}
\dot{\lambda} & =2\langle\dot{v}, A v\rangle+\langle v, \dot{A} v\rangle \\
& =2\langle\lambda v-A v-B v, A v\rangle+\langle v, \dot{A} v\rangle \\
& =-2\|\lambda v-A v\|^{2}+2\langle B v, \lambda v-A v\rangle+\langle v, \dot{A} v\rangle \\
& \leqslant\|B\|^{2}+\|\dot{A}\|-\|\lambda v-A v\|^{2} .
\end{aligned}
$$

Thus we have proved (19) for $s \in \Omega$.

Next we prove that $\Omega=[0, \infty)$. Following [2] we consider the function $\gamma: \Omega \rightarrow \mathbb{R}$ given by

$$
\gamma(s):=\log \|\xi(s)\| .
$$

Its derivative is $\dot{\gamma}=-\lambda$ and hence, by (19),

$$
\ddot{\gamma}(s) \geqslant-\|B(s)\|^{2}-\|\dot{A}(s)\| .
$$

Since $\xi$ is nonconstant, $\Omega \neq \emptyset$. Let $s_{1} \in \Omega$, suppose by contradiction that $\Omega \neq[0, \infty)$, and choose a real number $s_{2} \geqslant 0$ such that $s_{2} \notin \Omega$. Choose $c>0$ such that $\|B(s)\|^{2}+$ $\|\dot{A}(s)\| \leqslant c$ for every $s$ in the interval between $s_{1}$ and $s_{2}$. Assume first that $s_{2}>s_{1}$. Then $s_{2}$ may be chosen such that $\left[s_{1}, s_{2}\right) \subset \Omega$. By (20), we have

$$
\dot{\gamma}(s)=\dot{\gamma}\left(s_{1}\right)+\int_{s_{1}}^{s} \ddot{\gamma} \geqslant \dot{\gamma}\left(s_{1}\right)-c\left(s_{2}-s_{1}\right)=:-c^{\prime}
$$

and hence $\gamma(s)=\gamma\left(s_{1}\right)+\int_{s_{1}}^{s} \dot{\gamma} \geqslant \gamma\left(s_{1}\right)-c^{\prime}\left(s_{2}-s_{1}\right)$ for $s_{1} \leqslant s<s_{2}$. This implies that $\|\xi(s)\|=\mathrm{e}^{\gamma(s)}$ does not converge to zero as $s$ tends to $s_{2}$. Hence $s_{2} \in \Omega$ in contradiction to our assumption. Now suppose $s_{2}<s_{1}$. Then $s_{2}$ may be chosen such that $\left(s_{2}, s_{1}\right] \subset \Omega$. By (20), we have

$$
\dot{\gamma}(s)=\dot{\gamma}\left(s_{1}\right)-\int_{s}^{s_{1}} \ddot{\gamma} \leqslant \dot{\gamma}\left(s_{1}\right)+c\left(s_{1}-s_{2}\right)=: c^{\prime \prime}
$$


and hence $\gamma(s)=\gamma\left(s_{1}\right)-\int_{s}^{s_{1}} \dot{\gamma} \geqslant \gamma\left(s_{1}\right)-c^{\prime \prime}\left(s_{1}-s_{2}\right)$ for $s_{2}<s \leqslant s_{1}$. It follows again that $\|\xi(s)\|=\mathrm{e}^{\gamma(s)}$ does not converge to zero as $s$ tends to $s_{2}$ and so $s_{2} \in \Omega$. Thus we have proved that $\Omega=[0, \infty)$. Now suppose that

$$
N=-\dot{\gamma}(0)+\int_{0}^{\infty}\left(\|B(s)\|^{2}+\|\dot{A}(s)\|\right) d s<\infty .
$$

Then, by (20),

$$
\dot{\gamma}(s)=\dot{\gamma}(0)+\int_{0}^{s} \ddot{\gamma}(\sigma) d \sigma \geqslant-N
$$

and hence $\gamma(s) \geqslant \gamma(0)-N s$ for every $s \geqslant 0$. This implies

$$
\|\xi(s)\|=\mathrm{e}^{\gamma(s)} \geqslant \mathrm{e}^{\gamma(0)} \mathrm{e}^{-N s}=\mathrm{e}^{-N s}\|\xi(0)\|
$$

for every $s \geqslant 0$.

Lemma 3.4. - Assume that $A, B, \xi, v, \lambda$ satisfy the hypotheses of Lemma 3.3, let $\delta$ be the constant of Lemma 3.1, and $N$ be the constant of Lemma 3.3. Assume further that

$$
\int_{0}^{\infty}\left(\left\|A(s)-A_{\infty}\right\|+\|B(s)\|\right) d s<\infty .
$$

Then the limits

$$
\lambda_{\infty}=\lim _{s \rightarrow \infty} \lambda(s), \quad v_{\infty}=\lim _{s \rightarrow \infty} v(s)
$$

exist (the latter convergence is in $H$ ), $\delta \leqslant \lambda_{\infty} \leqslant N, v_{\infty} \in V$, and

$$
A_{\infty} v_{\infty}=\lambda_{\infty} v_{\infty} .
$$

Proof. - Consider the function

$$
\mu(s):=\lambda(s)+\int_{s}^{\infty}\left(\|B\|^{2}+\|\dot{A}\|\right) .
$$

By (17), we have

$$
\mu(0)=N, \quad \mu(s) \geqslant \lambda(s) \geqslant \delta \quad \text { for } s \geqslant s_{0} .
$$

Since $\dot{\mu}(s)=\dot{\lambda}(s)-\|B(s)\|^{2}-\|\dot{A}(s)\|$ it follows from (19) that

$$
\dot{\mu}(s)+\|A(s) v(s)-\lambda(s) v(s)\|^{2} \leqslant 0
$$

for every $s \geqslant 0$. Hence $\mu(s)$ converges to a positive real number

$$
\lambda_{\infty}:=\lim _{s \rightarrow \infty} \mu(s)=\lim _{s \rightarrow \infty} \lambda(s) .
$$


Since $\mu(0)=N$ and $\mu(s) \geqslant \delta$ for every $s \geqslant s_{0}$ we have

$$
\delta \leqslant \lambda_{\infty} \leqslant N
$$

We prove that $\lambda_{\infty}$ is an eigenvalue of $A_{\infty}$. Suppose, otherwise, that the operator $A_{\infty}-\lambda_{\infty}: V \rightarrow H$ is injective. The inclusion $V \hookrightarrow H$ is a compact operator and $A_{\infty}$ is bijective, and so $A_{\infty}-\lambda_{\infty}$ is a Fredholm operator of index zero. Hence $A_{\infty}-\lambda_{\infty}$ is bijective and hence, by the open mapping theorem, there exists a constant $c>0$ such that, for every $\eta \in V$,

$$
\|\eta\| \leqslant 3 c\left\|A_{\infty} \eta-\lambda_{\infty} \eta\right\|
$$

Choose $s_{\infty} \geqslant 0$ such that

$$
\left\|A(s)-A_{\infty}\right\| \leqslant c, \quad\left|\lambda(s)-\lambda_{\infty}\right| \leqslant c
$$

for $s \geqslant s_{\infty}$. Then

$$
1 \leqslant c\|A(s) v(s)-\lambda(s) v(s)\|
$$

and, by (22), $\dot{\mu}(s) \leqslant-1 / c^{2}$ for $s \geqslant s_{\infty}$. This contradicts the existence of the limit (23). Thus we have proved that $\lambda_{\infty}$ is an eigenvalue of $A_{\infty}$. Next we prove that

$$
\lim _{s \rightarrow \infty} \sigma(s)=0, \quad \sigma(s):=\left\|v(s)-\lambda_{\infty} A_{\infty}{ }^{-1} v(s)\right\|^{2} .
$$

Since $\langle v, \dot{v}\rangle=0$, the function $\sigma$ has a bounded derivative

$$
\dot{\sigma}=2 \lambda_{\infty}\left\langle\lambda_{\infty} A_{\infty}{ }^{-1} v-2 v, A_{\infty}{ }^{-1} \dot{v}\right\rangle .
$$

Now suppose, by contradiction, that $\sigma(s)$ does not converge to zero. Then there exists a sequence $s_{v} \rightarrow \infty$ and a constant $\varepsilon>0$ such that $\sigma\left(s_{v}\right)>3 \varepsilon$. Since $|\dot{\sigma}|$ is uniformly bounded, say by some constant $c>0$, we have

$$
s_{v}-\varepsilon / c \leqslant s \leqslant s_{v}+\varepsilon / c \Longrightarrow \sigma(s) \geqslant 2 \varepsilon .
$$

Hence

$$
\left\|A_{\infty} v(s)-\lambda_{\infty} v(s)\right\|^{2} \geqslant 2 \varepsilon /\left\|A_{\infty}{ }^{-1}\right\|^{2}
$$

for $s \in\left[s_{v}-\varepsilon / c, s_{v}+\varepsilon / c\right]$. Since $\left\|A(s)-A_{\infty}\right\|$ and $\left|\lambda(s)-\lambda_{\infty}\right|$ converge to zero as $s \rightarrow \infty$, it follows that

$$
\|A(s) v(s)-\lambda(s) v(s)\|^{2} \geqslant \varepsilon /\left\|A_{\infty}{ }^{-1}\right\|^{2}
$$

for $s \in\left[s_{v}-\varepsilon / c, s_{v}+\varepsilon / c\right]$ and $v$ sufficiently large. Hence it follows from (22) that $\mu(s)$ diverges to $-\infty$ as $s \rightarrow \infty$. This contradicts (23). Thus we have proved (24). Let

$$
E:=\operatorname{ker}\left(A_{\infty}-\lambda_{\infty}\right)
$$


and denote by $P: H \rightarrow E$ the orthogonal projection. Then there exists a constant $c>0$ such that, for every $\eta \in H$,

$$
\|\eta-P \eta\| \leqslant c\left\|\eta-\lambda_{\infty} A_{\infty}{ }^{-1} \eta\right\| .
$$

Hence, by (24),

$$
\lim _{s \rightarrow \infty}\|v(s)-P v(s)\|=0, \quad \lim _{s \rightarrow \infty}\|P v(s)\|=\lim _{s \rightarrow \infty}\langle v(s), P v(s)\rangle=1 .
$$

Now

$$
P \dot{\xi}(s)=-\lambda_{\infty} P \xi(s)+f(s), \quad f(s):=P\left(A_{\infty}-A(s)-B(s)\right) \xi(s) .
$$

By (25), there exists a constant $s_{1}>0$ such that $\|\xi(s)\| \leqslant 2\|P \xi(s)\|$ for $s \geqslant s_{1}$ and hence

$$
\|f(s)\| \leqslant 2\left(\left\|A_{\infty}-A(s)\right\|+\|B(s)\|\right)\|P \xi(s)\|
$$

for $s \geqslant s_{1}$. Consider the function

$$
w(s):=\frac{P \xi(s)}{\|P \xi(s)\|}=\frac{P v(s)}{\|P v(s)\|} .
$$

By (26), its derivative is

$$
\dot{w}=\frac{P \dot{\xi}}{\|P \xi\|}-\left\langle\frac{P \dot{\xi}}{\|P \xi\|}, w\right\rangle w=\frac{f}{\|P \xi\|}-\left\langle\frac{f}{\|P \xi\|}, w\right\rangle w .
$$

By (27), the derivative $\dot{w}$ is integrable. Hence $w(s)$ converges to an element $v_{\infty} \in E$ of norm one. Hence, by (25),

$$
v_{\infty}=\lim _{s \rightarrow \infty} w(s)=\lim _{s \rightarrow \infty} P v(s)=\lim _{s \rightarrow \infty} v(s) .
$$

This proves the lemma.

LEMmA 3.5. - Assume the situation of Lemma 3.4 and suppose that

$$
\int_{0}^{\infty} \int_{s}^{\infty}\left(\|B(\sigma)\|^{2}+\|\dot{A}(\sigma)\|\right) d \sigma d s<\infty .
$$

Then $\lambda-\lambda_{\infty}$ is integrable,

$$
\lim _{s \rightarrow \infty} \mathrm{e}^{\lambda \infty s}\|\xi(s)\|=c_{\infty}:=\mathrm{e}^{\int_{0}^{\infty}(\lambda \infty-\lambda)}\|\xi(0)\|,
$$

and

$$
\left\|\xi(s)-\mathrm{e}^{-\lambda_{\infty} s} c_{\infty} v_{\infty}\right\|=\mathrm{o}\left(\mathrm{e}^{-\lambda_{\infty} s}\right)
$$

as $s$ tends to $\infty$. 
Proof. - Consider the function

$$
\rho(s):=\frac{1}{2}\|v(s)-P v(s)\|^{2} .
$$

Its derivative is

$$
\begin{aligned}
\dot{\rho} & =\langle v-P v, \dot{v}\rangle \\
& =\langle v-P v, \lambda v-A v-B v\rangle \\
& =\langle-P v, \lambda v-A v-B v\rangle \\
& =\left(\lambda_{\infty}-\lambda\right)\langle P v, v\rangle+\left\langle P v, A v-A_{\infty} v+B v\right\rangle \\
& \leqslant\left\|A-A_{\infty}\right\|+\|B\|-\left(\lambda-\lambda_{\infty}\right)\langle P v, v\rangle \\
& \leqslant g-f\langle v, P v\rangle,
\end{aligned}
$$

where

$$
f:=\mu-\lambda_{\infty},
$$

$\mu$ is given by (21), and

$$
g(s):=\left\|A(s)-A_{\infty}\right\|+\|B(s)\|+\int_{s}^{\infty}\left(\|B\|^{2}+\|\dot{A}\|\right) .
$$

By (25), there exists a positive real number $s_{2}$ such that $\langle v(s), P v(s)\rangle \geqslant 1 / 2$ for every $s \geqslant s_{2}$. Moreover, since $\mu$ is decreasing with limit $\lambda_{\infty}$, it follows that $f$ is positive. Hence, for $s^{\prime} \geqslant s \geqslant s_{2}$,

$$
\int_{s}^{s^{\prime}} f \leqslant 2 \int_{s}^{s^{\prime}} f\langle v, P v\rangle \leqslant 2 \int_{s}^{s^{\prime}}(g-\dot{\rho}) \leqslant 2\left(\rho(s)+\int_{s}^{\infty} g\right) .
$$

Since $g$ is integrable it follows that $f$ is integrable. By assumption and (21), $\mu-\lambda$ is integrable, and hence so is $\lambda_{\infty}-\lambda=\mu-\lambda-f$. Next we observe that the function $c(s):=\mathrm{e}^{\lambda \infty s}\|\xi(s)\|$ satisfies the differential equation

$$
\dot{c}(s)=\lambda_{\infty} c(s)-\mathrm{e}^{\lambda_{\infty} s} \frac{\langle\xi(s), A(s) \xi(s)\rangle}{\|\xi(s)\|}=\left(\lambda_{\infty}-\lambda(s)\right) c(s) .
$$

Hence

$$
c(s)=\mathrm{e}^{\lambda \infty s}\|\xi(s)\|=\mathrm{e}^{\int_{0}^{s}\left(\lambda_{\infty}-\lambda\right)}\|\xi(0)\|
$$

and hence $c(s)$ converges to $c_{\infty}$ as $s$ tends to $\infty$. This proves (28). To prove (29) note that

$$
\lim _{s \rightarrow \infty} \mathrm{e}^{\lambda_{\infty} s} \xi(s)=\lim _{s \rightarrow \infty} c(s) v(s)=c_{\infty} v_{\infty}
$$

where the convergence is in $H$. 
Lemma 3.6. - Assume the situation of Lemma 3.5 and suppose that there exist positive constants $C$ and $\varepsilon$ such that

$$
\left\|A(s)-A_{\infty}\right\|+\|B(s)\|+\|\dot{A}(s)\|+\|\dot{B}(s)\| \leqslant C \mathrm{e}^{-\varepsilon s} .
$$

Then there exist positive constants $c$ and $\delta$ such that

$$
\left\|\xi(s)-\mathrm{e}^{-\lambda \infty s} c_{\infty} v_{\infty}\right\| \leqslant c \mathrm{e}^{-\left(\lambda_{\infty}+\delta\right) s} .
$$

Proof. - Since $A_{\infty}-\lambda_{\infty}: V \rightarrow H$ is a Fredholm operator its kernel $E$ is finite dimensional. Think of $E$ as a subspace of $H$ and denote its orthogonal complement by

$$
H_{1}:=E^{\perp}, \quad V_{1}:=V \cap H_{1} .
$$

As above, let $P: H \rightarrow E$ denote the orthogonal projection and consider the operators $A_{1}(s): V_{1} \rightarrow H_{1}$ and $B_{1}(s): H_{1} \rightarrow H_{1}$ defined by

$$
A_{1}(s):=(\mathbb{1}-P) A(s)(\mathbb{1}-P)-\lambda_{\infty}, \quad B_{1}(s):=(\mathbb{1}-P) B(s)(\mathbb{1}-P) .
$$

Define $\xi_{1}:[0, \infty) \rightarrow V_{1}$ and $\eta_{1}:[0, \infty) \rightarrow H_{1}$ by

$$
\xi_{1}(s):=\mathrm{e}^{\lambda_{\infty} s}(\mathbb{1}-P) \xi(s),
$$

and

$$
\eta_{1}(s):=\mathrm{e}^{\lambda_{\infty} s}(\mathbb{1}-P)(A(s)+B(s)) P \xi(s) .
$$

Then $\xi_{1}$ and $\eta_{1}$ are continuously differentiable as functions from $[0, \infty)$ to $H_{1}$ and

$$
\dot{\xi}_{1}+A_{1} \xi_{1}+B_{1} \xi_{1}+\eta_{1}=0
$$

The derivative of $\eta_{1}$ is

$$
\begin{aligned}
\dot{\eta}_{1}(s)= & \mathrm{e}^{\lambda_{\infty} s}(\mathbb{1}-P)(\dot{A}(s)+\dot{B}(s)) P \xi(s) \\
& -\mathrm{e}^{\lambda_{\infty} s}(\mathbb{1}-P)(A(s)+B(s)) P\left(A(s)-\lambda_{\infty}+B(s)\right) \xi(s) \\
= & \mathrm{e}^{\lambda_{\infty} s}(\mathbb{1}-P)(\dot{A}(s)+\dot{B}(s)) P \xi(s) \\
& -\mathrm{e}^{\lambda_{\infty} s}(\mathbb{1}-P)\left(A(s)-A_{\infty}+B(s)\right) P\left(A(s)-A_{\infty}+B(s)\right) \xi(s) .
\end{aligned}
$$

Hence, by (28) and (33), there is a constant $C^{\prime}>0$ such that, for every $s \geqslant 0$,

$$
\left\|\eta_{1}(s)\right\|+\left\|\dot{\eta}_{1}(s)\right\| \leqslant C^{\prime} \mathrm{e}^{-\varepsilon s} .
$$

Hence $\xi_{1}$ and $\eta_{1}$ satisfy the hypotheses of Lemma 3.1. Hence there exist constants $c>0$ and $\delta \in(0, \varepsilon)$ such that, for every $s \geqslant 0$,

$$
\|v(s)-P v(s)\|=\frac{\left\|\xi_{1}(s)\right\|}{\mathrm{e}^{\lambda \infty s}\|\xi(s)\|} \leqslant c \mathrm{e}^{-\delta s} .
$$


Moreover, it follows from (27) and (33) that there exist positive constants $c^{\prime}$ and $s^{\prime}$ such that, for every $s \geqslant s^{\prime}$,

$$
\left\|\frac{P \xi(s)}{\|P \xi(s)\|}-v_{\infty}\right\|=\left\|w(s)-v_{\infty}\right\| \leqslant \int_{s}^{\infty}\|\dot{w}\| \leqslant c^{\prime} \mathrm{e}^{-\varepsilon s} .
$$

Hence

$$
\begin{aligned}
\left\|v(s)-v_{\infty}\right\| & \leqslant\|v(s)-P v(s)\|+\left\|P v(s)-\frac{P v(s)}{\|P v(s)\|}\right\|+\left\|\frac{P v(s)}{\|P v(s)\|}-v_{\infty}\right\| \\
& =\|v(s)-P v(s)\|+1-\|P v(s)\|+\left\|\frac{P \xi(s)}{\|P \xi(s)\|}-v_{\infty}\right\| \\
& \leqslant 2\|v(s)-P v(s)\|+\left\|\frac{P \xi(s)}{\|P \xi(s)\|}-v_{\infty}\right\| \\
& \leqslant\left(2 c+c^{\prime}\right) \mathrm{e}^{-\delta s}
\end{aligned}
$$

for every $s \geqslant s^{\prime}$. Now let $g:[0, \infty) \rightarrow[0, \infty)$ be given by (30). Then, by (33), there is a constant $c^{\prime \prime}>0$ such that, for every $s \geqslant 0$,

$$
\int_{s}^{\infty} g(s) d s \leqslant c^{\prime \prime} \mathrm{e}^{-\varepsilon s} .
$$

By (31) and (34), there is a constant $c^{\prime \prime \prime}>0$ such that, for every $s \geqslant 0$,

$$
\int_{s}^{\infty}\left(\mu-\lambda_{\infty}\right)=\int_{s}^{\infty} f \leqslant\|v(s)-P v(s)\|^{2}+2 \int_{s}^{\infty} g \leqslant c^{\prime \prime \prime} \mathrm{e}^{-\delta s} .
$$

By (21) and (33), there is a constant $c^{\prime \prime \prime \prime \prime}>0$ such that, for every $s \geqslant 0$,

$$
\int_{s}^{\infty}\left|\lambda-\lambda_{\infty}\right| \leqslant \int_{s}^{\infty}|\lambda-\mu|+\int_{s}^{\infty}\left|\mu-\lambda_{\infty}\right| \leqslant c^{\prime \prime \prime \prime} \mathrm{e}^{-\delta s} .
$$

By (32), $c(s)=\mathrm{e}^{\lambda \infty s}\|\xi(s)\|$ converges exponentially to $c_{\infty}$ and hence the function $\mathrm{e}^{\lambda_{\infty} s} \xi(s)=c(s) v(s)$ converges exponentially to $c_{\infty} v_{\infty}$.

\section{Proofs of Theorems $A$ and $B$}

Proof of Theorem A. - It is immediate that (III) implies (I); in fact, if $u$ satisfies (III) then

$$
E(u) \leqslant \frac{c}{\varepsilon}
$$


We prove that (I) implies (II). Assume, by contradiction, that there exist sequences $s_{v} \rightarrow \infty$ and $t_{v} \in[0,1]$ and a constant $\delta>0$ such that

$$
\left|\partial_{s} u\left(s_{v}, t_{v}\right)\right| \geqslant \delta
$$

for every $v$. Consider the sequence

$$
u_{v}(s, t):=u\left(s_{v}+s, t\right) .
$$

By (I) and Lemma B.3, there exist constants $s_{0}>0$ and $c_{1}>0$ such that, for every $s \in \mathbb{R}$ and every $t \in[0,1]$,

$$
s \geqslant s_{0} \Longrightarrow\left|\partial_{s} u(s, t)\right| \leqslant c_{1} .
$$

Hence, by Lemma C.3, there exist constants $v_{0}>0$ and $c_{2}>0$ such that

$$
v \geqslant v_{0} \Longrightarrow\left\|u_{v}\right\|_{C^{2}([-1,1] \times[0,1])} \leqslant c_{2} .
$$

By the Arzéla-Ascoli theorem, $u_{v}$ has a $C^{1}$-convergent subsequence, still denoted by $u_{v}$. Since $E(u)=0$, the limit function is independent of the $s$-variable. Hence

$$
\lim _{\nu \rightarrow \infty}\left\|\partial_{s} u_{\nu}\right\|_{C^{0}([-1,1] \times[0,1])}=0 .
$$

This contradicts (35). Thus we have proved that the second limit in (II) exists. Since $\partial_{s} u+J_{t}(u) \partial_{t} u=0$ it follows that $\partial_{t} u$ converges to zero uniformly. Hence the length function $s \mapsto \int_{0}^{1}\left|\partial_{t} u(s, t)\right| d t$ converges to zero. Hence it follows from the boundary condition that $u(s, t)$ converges to an intersection point of $L_{0}$ and $L_{1}$ as $s$ tends to $\infty$. This proves (II).

Before proving (II) implies (III) we first prove that (II) implies

$$
\lim _{s \rightarrow \infty}\left\|\partial_{s} u\right\|_{C^{k}([s, \infty) \times[0,1])}=0
$$

for every $k$. If (II) holds then $\sup _{s, t}\left|\partial_{s} u(s, t)\right|<\infty$. Hence, by Lemma C.3 and the fact that $J$ is independent of $s$, it follows that, for every $k$,

$$
\left\|\partial_{s} u\right\|_{C^{k}([0, \infty) \times[0,1])}<\infty .
$$

Now suppose, by contradiction, that there exist a $\delta>0$, an integer $k \geqslant 0$, and a sequence $s_{v} \rightarrow \infty$ such that

$$
\left\|\partial_{s} u\right\|_{C^{k}\left(\left[s_{v}-1, s_{v}+1\right] \times[0,1]\right)} \geqslant \delta .
$$

Since $\partial_{s} u$ satisfies a uniform $C^{k+1}$-bound, it follows that the sequence

$$
\xi_{v}(s, t):=\partial_{s} u\left(s+s_{v}, t\right)
$$

has a $C^{k}$-convergent subsequence. Since $\partial_{s} u$ converges to zero in the sup-norm the limit is zero. Hence the subsequence of $\xi_{v}$ converges to zero in the $C^{k}$-norm, a contradiction. Thus we have proved (36). 
We prove that (II) implies (III). If (II) holds we may assume, without loss of generality, that $u(s, t) \in U$ for every $s \geqslant 0$ and every $t \in[0,1]$, where $U \subset M$ is the neighbourhood of $p$ introduced in Lemma 2.1. For $q \in U$ and $t \in[0,1]$ let $\Phi_{t}(q): \mathbb{R}^{2 n} \rightarrow T_{q} M$ be the trivialization of Lemma 2.1 and, for $s \geqslant 0$ and $0 \leqslant t \leqslant 1$, define $\xi(s, t) \in \mathbb{R}^{2 n}$ by

$$
\xi(s, t):=\Phi_{t}(u(s, t))^{-1} \partial_{s} u(s, t) .
$$

Since $D_{u} \partial_{s} u=0$, it follows from (4) that

$$
\partial_{s} \xi+J_{0} \partial_{t} \xi+S \xi=0 .
$$

Define $\xi:[0, \infty) \rightarrow V$ by $\xi(s)(t):=\xi(s, t)$. This function is smooth and, by (38), it satisfies

$$
\dot{\xi}(s)+A(s) \xi(s)+B(s) \xi(s)=0,
$$

where $A(s)$ and $B(s)$ are defined by (8) and (9). By (36) and Lemma 2.3, $A(s)$ and $B(s)$ satisfy the hypotheses of Lemma 3.1. Hence there exist positive constants $c_{0}$ and $\varepsilon$ such that, for every $s \geqslant 0$,

$$
\int_{0}^{1}|\xi(s, t)|^{2} d t \leqslant c_{0} \mathrm{e}^{-2 \varepsilon s} .
$$

Now consider Eq. (38). By Lemma C.1, there exist, for each integer $k \geqslant 0$, constants $c_{k}$ and $c_{k}^{\prime}$ such that, for every $s \geqslant 1$,

$$
\begin{aligned}
\|\xi\|_{W^{k, 2}([s, \infty) \times[0,1])} & \leqslant c_{k}\left(\|S \xi\|_{W^{k-1,2([s-1, \infty) \times[0,1])}}+\|\xi\|_{W^{k-1,2}([s-1, \infty) \times[0,1])}\right) \\
& \leqslant c_{k}^{\prime}\|\xi\|_{W^{k-1,2([s-1, \infty) \times[0,1])}} .
\end{aligned}
$$

Here the last inequality uses the fact that, by (36) and Lemma 2.2, the function $S$ satisfies a uniform $C^{k-1}$-bound. Hence, by induction,

$$
\|\xi\|_{W^{k, 2}([s, \infty) \times[0,1])} \leqslant c_{k}^{\prime \prime}\|\xi\|_{L^{2}([s-k, \infty) \times[0,1])} \leqslant c_{k}^{\prime \prime} \sqrt{\frac{c_{0}}{2 \varepsilon}} \mathrm{e}^{-\varepsilon(s-k)}
$$

for $s \geqslant s_{0}+k$. The last inequality follows from (40). Combining this with (36) we obtain that $\partial_{s} u=\Phi_{t}(u) \xi$ converges to zero exponentially in the $C^{\infty}$ topology, as required.

Proof of Theorem B. - Let $u \in \mathcal{S}$ be nonconstant and continue the notation of the proof of Theorem A. In particular, $\xi(s, t) \in \mathbb{R}^{2 n}$ is defined by (37) and satisfies (38), (39), and (40). By (III) in Theorem A and Lemma 2.3, the operators $A(s)$ and $B(s)$ satisfy the hypotheses of Lemma 3.6. Hence there exist an eigenvalue $\lambda_{\infty}>0$, a nonzero eigenfunction $v_{\infty} \in \operatorname{ker}\left(A_{\infty}-\lambda_{\infty}\right)$, and constants $c>0$ and $\delta \in(0, \varepsilon)$ such that, for every $s \geqslant 0$,

$$
\int_{0}^{1}\left|\mathrm{e}^{\lambda_{\infty} s} \xi(s, t)-v_{\infty}(t)\right|^{2} d t \leqslant c \mathrm{e}^{-2 \delta s} .
$$


Abbreviate

$$
\zeta(s, t):=\mathrm{e}^{\lambda \infty s} \xi(s, t)-v_{\infty}(t) .
$$

We prove by induction that, for every integer $k \geqslant 0$, there exists a constant $c_{k}>0$ such that, for every $s \geqslant 0$,

$$
\|\zeta\|_{W^{k, 2}([s, \infty) \times[0,1])} \leqslant c_{k} \mathrm{e}^{-\delta s} .
$$

For $k=0$ this follows from (41). Assume, by induction, that (43) has been established for some integer $k \geqslant 0$. Note that $\zeta$ satisfies the partial differential equation

$$
\partial_{s} \zeta+J_{0} \partial_{t} \zeta=\eta
$$

where

$$
\eta(s, t):=\mathrm{e}^{\lambda_{\infty} s}\left(S_{\infty}(t)-S(s, t)\right) \xi(s, t)-\left(S_{\infty}(t)-\lambda_{\infty}\right) \zeta(s, t),
$$

and the boundary condition

$$
\zeta(s, 0), \zeta(s, 1) \in \mathbb{R}^{n} \times\{0\} .
$$

By (III) in Theorem A and (7) in Lemma 2.2, there exists a constant $c_{k}^{\prime}>0$ such that

$$
\left\|S-S_{\infty}\right\|_{C^{k}([s, \infty) \times[0,1])} \leqslant c_{k}^{\prime} \mathrm{e}^{-\delta s}
$$

for every $s \geqslant 0$. Hence it follows from the induction hypothesis that

$$
\|\eta\|_{W^{k, 2}([s, \infty) \times[0,1])} \leqslant c_{k}^{\prime \prime} \mathrm{e}^{-\delta s}
$$

for every $s \geqslant 0$ and some constant $c_{k}^{\prime \prime}$. Hence it follows from Lemma C.1 that (43) holds with $k$ replaced by $k+1$.

With (43) established, it follows from the Sobolev embedding theorem that $\mathrm{e}^{\lambda_{\infty} s} \xi(s, t)$ converges uniformly and exponentially with all derivatives to $v_{\infty}(t)$ as $s$ tends to $\infty$. Consider the function $u$ in $t$-dependent local coordinates $\varphi_{t}: U \rightarrow \mathbb{R}^{2 n}$ near $p$ such that

$$
\varphi_{t}(p)=0, \quad d \varphi_{t}(p)=\Phi_{t}(p)^{-1} .
$$

Then the matrix function $\Psi_{t}: U \rightarrow \mathbb{R}^{2 n \times 2 n}$, defined by

$$
\Psi_{t}(q):=d \varphi_{t}(q) \Phi_{t}(q),
$$

satisfies $\Psi_{t}(p)=\mathbb{1}$. Moreover, $\partial_{s}\left(\varphi_{t} \circ u\right)=\Psi_{t}(u) \xi$ and hence, by (42),

$$
\partial_{s} \varphi_{t}(u(s, t))=\mathrm{e}^{-\lambda \infty s} v_{\infty}(t)+R(s, t),
$$

and hence

$$
\varphi_{t}(u(s, t))=-\frac{1}{\lambda_{\infty}} \mathrm{e}^{-\lambda_{\infty} s} v_{\infty}(t)-\int_{s}^{\infty} R(\sigma, t) d \sigma,
$$


where

$$
R(s, t):=\mathrm{e}^{-\lambda_{\infty} s} \Psi_{t}(u(s, t)) \zeta(s, t)+\mathrm{e}^{-\lambda_{\infty} s}\left(\Psi_{t}(u(s, t))-\mathbb{1}\right) v_{\infty}(t) .
$$

It follows from (III) in Theorem A and (43) that, for every integer $k \geqslant 0$, there exists a constant $c_{k}>0$ such that, for every $s \geqslant 0$,

$$
\|R\|_{C^{k}([s, \infty) \times[0,1])} \leqslant c_{k} \mathrm{e}^{-\left(\lambda_{\infty}+\delta\right) s} .
$$

Since $v_{\infty}$ is a nonzero eigenfunction of $A_{\infty}$ it follows from (44) that $v_{\infty}(t) \neq 0$ for all $t$. Hence there exist positive constants $c$ and $s_{0}$ such that

$$
\frac{1}{c} \mathrm{e}^{-\lambda_{\infty} s} \leqslant\left|\partial_{s} u(s, t)\right| \leqslant c \mathrm{e}^{\lambda_{\infty} s}
$$

for $s \geqslant s_{0}$. Now take

$$
\lambda:=\lambda_{\infty}, \quad v(t):=\Phi_{t}(p) v_{\infty}(t) .
$$

Then it follows from (45) and (46) that $u$ has the required asymptotic behaviour. This proves Theorem B.

\section{Appendix A. The Heinz trick}

Let

$$
\Delta=\frac{\partial^{2}}{\partial x_{1}{ }^{2}}+\cdots+\frac{\partial^{2}}{\partial x_{n}{ }^{2}}
$$

denote the standard Laplacian on $\mathbb{R}^{n}$. We write

$$
B_{r}(x)=\left\{\xi \in \mathbb{R}^{n}|| \xi-x \mid<r\right\}
$$

and abbreviate $B_{r}=B_{r}(0)$. The following Lemma is a generalization of the mean value inequality for subharmonic functions. A version of this estimate was proved by Uhlenbeck [19] and used for the proof of the removable singularity theorem for YangMills connections. The proof below uses a classical trick by E. Heinz, which was explained to us by Mario Micallef. The second author used a similar inequality in [16].

LEMMA A.1. - For every $\lambda>1$ there exists a constant $\mu=\mu(\lambda, n)>0$ such that the following holds. If $w: B_{r} \rightarrow \mathbb{R}$ is a bounded $C^{2}$-function that satisfies the inequalities

$$
\Delta w \geqslant-a-b w^{(n+2) / n}, \quad w \geqslant 0, \quad \int_{B_{r}} w<\frac{\mu}{b^{n / 2}}
$$

for some constants $a \geqslant 0$ and $b \geqslant 0$ then

$$
w(0) \leqslant \frac{a r^{2}}{2 n+4}+\frac{\lambda}{\operatorname{Vol}\left(B_{r}\right)} \int_{B_{r}} w .
$$


Remark A.2. - If $b=0$ then the last condition in (A.1) is vacuous and (A.2) holds with $\lambda=1$. In this case the inequality is sharp, i.e. in (A.2) equality holds with $\lambda=1$ whenever $\Delta w=-a$. (See step 1 of the proof.)

Remark A.3. - The proof shows that the constant $\mu$ can be chosen as

$$
\mu=(2 n+4)^{n / 2}(1-\delta) \delta^{\left(n^{2}+3 n+2\right) / 2} \operatorname{Vol}\left(B_{1}\right),
$$

where $\delta \in(0,1)$ is given by $\lambda=(1-\delta)^{-n-1}$. In particular, with $\delta=1 / 2$ and $n=2$,

$$
\lambda=8, \quad \mu=\frac{\pi}{16} .
$$

Proof of Lemma A.1. - The proof consists of five steps.

Step 1: The lemma holds with $b=0$.

In this case the third inequality in (A.1) is automatically satisfied and (A.2) with $\lambda=1$ is the mean value inequality for the subharmonic function

$$
\tilde{w}(x):=w(x)+\frac{a|x|^{2}}{2 n} .
$$

Step 2: It suffices to prove the lemma for $r=1$.

Suppose that $w: B_{r} \rightarrow \mathbb{R}$ satisfies (A.1) and define $\tilde{w}: B_{1} \rightarrow \mathbb{R}$ and $\tilde{a}, \tilde{b} \in \mathbb{R}$ by

$$
\tilde{w}(z):=w(r z), \quad \tilde{a}:=a r^{2}, \quad \tilde{b}:=b r^{2} .
$$

Then

$$
\Delta \tilde{w} \geqslant-\tilde{a}-\tilde{b} \tilde{w}^{(n+2) / n}
$$

and

$$
\int_{B_{1}} \tilde{w}=\frac{1}{r^{n}} \int_{B_{r}} w \leqslant \frac{\mu}{r^{n} b^{n / 2}}=\frac{\mu}{\tilde{b}^{n / 2}} .
$$

Hence, assuming the lemma for $r=1$, we obtain

$$
w(0)=\tilde{w}(0) \leqslant \frac{\tilde{a}}{2 n+4}+\frac{\lambda}{\operatorname{Vol}\left(B_{1}\right)} \int_{B_{1}} \tilde{w}=\frac{a r^{2}}{2 n+4}+\frac{\lambda}{\operatorname{Vol}\left(B_{r}\right)} \int_{B_{r}} w .
$$

Step 3: It suffices to prove the lemma for $b=1$.

Suppose that $w: B_{r} \rightarrow \mathbb{R}$ satisfies (A.1) and define $\tilde{w}: B_{1} \rightarrow \mathbb{R}$ and $\tilde{a} \in \mathbb{R}$ by

$$
\tilde{w}(z):=b^{n / 2} w(z), \quad \tilde{a}:=b^{n / 2} a .
$$

Then

$$
\Delta \tilde{w} \geqslant-\tilde{a}-\tilde{w}^{(n+2) / n}
$$


and

$$
\int_{B_{r}} \tilde{w}=b^{n / 2} \int_{B_{r}} w \leqslant \mu .
$$

Hence, assuming the lemma for $b=1$, we obtain

$$
w(0)=b^{-n / 2} \tilde{w}(0) \leqslant \frac{b^{-n / 2} \tilde{a}}{2 n+4}+\frac{b^{-n / 2} \lambda}{\operatorname{Vol}\left(B_{r}\right)} \int_{B_{r}} \tilde{w}=\frac{a}{2 n+4}+\frac{\lambda}{\operatorname{Vol}\left(B_{r}\right)} \int_{B_{r}} w .
$$

Step 4 (The Heinz trick): Assume $b=r=1$ and define $f:[0,1] \rightarrow \mathbb{R}$ by

$$
f(\rho)=(1-\rho)^{n} \sup _{B_{\rho}} w
$$

for $0 \leqslant \rho \leqslant 1$. Since $f(1)=0$ and $f$ is nonegative, there exist $\rho^{*} \in[0,1)$ and $x^{*} \in B_{\rho^{*}}$ such that

$$
f\left(\rho^{*}\right)=\max _{0 \leqslant \rho \leqslant 1} f(\rho), \quad c:=w\left(x^{*}\right)=\sup _{B_{\rho^{*}}} w
$$

Denote

$$
\varepsilon:=(1-\delta)\left(1-\rho^{*}\right)
$$

Then, for $0 \leqslant \rho \leqslant \varepsilon$,

$$
c \leqslant \frac{a \rho^{2}}{2 n+4}+\frac{c^{(n+2) / n} \rho^{2}}{(2 n+4) \delta^{n+2}}+\frac{1}{\rho^{n} \operatorname{Vol}\left(B_{1}\right)} \int_{B_{1}} w .
$$

To see this, note first that

$$
\sup _{B_{\varepsilon}\left(x^{*}\right)} w \leqslant \sup _{B_{\rho^{*}+\varepsilon}} w=\frac{f\left(\rho^{*}+\varepsilon\right)}{\left(1-\rho^{*}-\varepsilon\right)^{n}}=\frac{f\left(\rho^{*}+\varepsilon\right)}{\delta^{n}\left(1-\rho^{*}\right)^{n}} \leqslant \frac{f\left(\rho^{*}\right)}{\delta^{n}\left(1-\rho^{*}\right)^{n}}=\frac{c}{\delta^{n}} .
$$

Hence

$$
\Delta w \geqslant-a-w^{(n+2) / n} \geqslant-a-\frac{c^{(n+2) / n}}{\delta^{n+2}}
$$

in $B_{\varepsilon}\left(x^{*}\right)$ and so (A.3) follows from step 1 with $r=\rho \leqslant \varepsilon$ and $a$ replaced by $a+c^{(n+2) / n} \delta^{-n-2}$.

Step 5: The lemma holds for $r=1$ and $b=1$.

If $(2 n+4) c \leqslant a$ then $w(0) \leqslant c \leqslant a /(2 n+4)$ and this implies (A.2). Hence we may assume that

$$
a \leqslant(2 n+4) c
$$

Next we prove that

$$
\frac{c^{2 / n} \varepsilon^{2}}{(2 n+4) \delta^{n+2}}<\delta
$$


Suppose otherwise that $\varepsilon^{2} \geqslant(2 n+4) \delta^{n+3} / c^{2 / n}$. Then, in (A.3), we can choose

$$
\rho:=\sqrt{\frac{(2 n+4) \delta^{n+3}}{c^{2 / n}}} \leqslant \varepsilon
$$

and obtain

$$
\begin{aligned}
c & \leqslant \frac{a \rho^{2}}{2 n+4}+\frac{c^{(n+2) / n} \rho^{2}}{(2 n+4) \delta^{n+2}}+\frac{1}{\rho^{n} \operatorname{Vol}\left(B_{1}\right)} \int_{B_{1}} w \\
& =\frac{a \delta^{n+3}}{c^{2 / n}}+\delta c+\left(\frac{c^{2 / n}}{(2 n+4) \delta^{n+3}}\right)^{n / 2} \frac{1}{\operatorname{Vol}\left(B_{1}\right)} \int_{B_{1}} w \\
& \leqslant \frac{(2 n+4) \delta^{n+3} c}{c^{2 / n}}+\delta c+\left(\frac{c^{2 / n}}{(2 n+4) \delta^{n+3}}\right)^{n / 2} \frac{1}{\operatorname{Vol}\left(B_{1}\right)} \int_{B_{1}} w \\
& \leqslant(1-\delta)^{2} c+\delta c+c\left(\frac{1}{(2 n+4) \delta^{n+3}}\right)^{n / 2} \frac{1}{\operatorname{Vol}\left(B_{1}\right)} \int_{B_{1}} w \\
& =c-\delta(1-\delta) c+c\left(\frac{1}{(2 n+4) \delta^{n+3}}\right)^{n / 2} \frac{1}{\operatorname{Vol}\left(B_{1}\right)} \int_{B_{1}} w .
\end{aligned}
$$

Here the third inequality uses $a \leqslant(2 n+4) c$ and the fourth inequality follows from the fact that $(2 n+4) \delta^{n+3} / c^{2 / n} \leqslant \varepsilon^{2} \leqslant(1-\delta)^{2}$. It follows that

$$
\delta(1-\delta)\left((2 n+4) \delta^{n+3}\right)^{n / 2} \operatorname{Vol}\left(B_{1}\right) \leqslant \int_{B_{1}} w
$$

But the left hand side equals $\mu$ (see Remark A.3) and so this contradicts (A.1). Hence (A.4) must have been true.

Now consider (A.3) with $\rho=\varepsilon$ to obtain

$$
\begin{aligned}
c & \leqslant \frac{a \varepsilon^{2}}{2 n+4}+\frac{c^{(n+2) / n} \varepsilon^{2}}{(2 n+4) \delta^{n+2}}+\frac{1}{\varepsilon^{n} \operatorname{Vol}\left(B_{1}\right)} \int_{B_{1}} w \\
& \leqslant \frac{a(1-\delta)^{n+2}}{(2 n+4) \varepsilon^{n}}+\delta c+\frac{1}{\varepsilon^{n} \operatorname{Vol}\left(B_{1}\right)} \int_{B_{1}} w .
\end{aligned}
$$

The last inequality uses $\varepsilon \leqslant 1-\delta$ and (A.4). Multiplying by $\varepsilon^{n}$ gives

$$
(1-\delta) c \varepsilon^{n} \leqslant \frac{a(1-\delta)^{n+2}}{2 n+4}+\frac{1}{\operatorname{Vol}\left(B_{1}\right)} \int_{B_{1}} w
$$


Hence

$$
w(0)=f(0) \leqslant f\left(\rho^{*}\right)=\left(1-\rho^{*}\right)^{n} c=\frac{c \varepsilon^{n}}{(1-\delta)^{n}} \leqslant \frac{a}{2 n+4}+\frac{(1-\delta)^{-n-1}}{\operatorname{Vol}\left(B_{1}\right)} \int_{B_{1}} w .
$$

This proves the lemma in the case $r=b=1$.

\section{Appendix B. Apriori estimates}

Throughout this section $(M, g)$ is a closed Riemannian manifold and $L \subset M$ is a closed submanifold. Denote by $\mathcal{J}(M, L)$ the space of all almost complex structures on $M$ for which $L$ is totally real. Denote

$$
\mathbb{H}=\{z \in \mathbb{C} \mid \operatorname{Im} z \geqslant 0\}, \quad H_{r}\left(z_{0}\right):=\left\{z \in \mathbb{H}|| z-z_{0} \mid<r\right\} .
$$

Note that $\pi r^{2} / 2 \leqslant \operatorname{area}\left(H_{r}\left(z_{0}\right)\right) \leqslant \pi r^{2}$.

LEMMA B.1. - For every $J \in \mathcal{J}(M, L)$ there exist constants $\delta>0$ and $c>0$ such that the following holds for every $r>0$, every $z_{0} \in \mathbb{H}$, and every smooth map $u: H_{r}\left(z_{0}\right) \rightarrow M$. If $u$ satisfies the boundary value problem

$$
\partial_{s} u+J(u) \partial_{t} u=0, \quad u\left(H_{r}\left(z_{0}\right) \cap \mathbb{R}\right) \subset L,
$$

then

$$
\int_{H_{r}\left(z_{0}\right)}\left|\partial_{s} u\right|^{2}<\delta \Longrightarrow\left|\partial_{s} u\left(z_{0}\right)\right|^{2} \leqslant \frac{c}{r^{2}} \int_{H_{r}\left(z_{0}\right)}\left|\partial_{s} u\right|^{2} .
$$

Proof. - The assertion is independent of the choice of the metric. Hence we may assume that $g$ satisfies the conditions (i), (ii), and (iii) of Lemma D.1. Let $\nabla$ denote the Levi-Civita connection of $g$ and $R \in \Omega^{2}(\operatorname{End}(T M))$ denote the curvature tensor. Abbreviate

$$
\xi:=\partial_{s} u, \quad \eta:=\partial_{t} u, \quad w:=\frac{1}{2}|\xi|^{2}=\frac{1}{2}|\eta|^{2},
$$

and $\Delta:=\partial_{s}{ }^{2}+\partial_{t}{ }^{2}$. (That $|\xi|=|\eta|$ follows from condition (i) in Lemma D.1.) Then

$$
\Delta w=\left|\nabla_{s} \xi\right|^{2}+\left|\nabla_{t} \xi\right|^{2}+\left\langle\xi, \nabla_{s} \nabla_{s} \xi+\nabla_{t} \nabla_{t} \xi\right\rangle
$$

Since $\nabla_{s} \eta=\nabla_{t} \xi$ we have

$$
\nabla_{s} \xi+\nabla_{t} \eta=\nabla_{t}(J \xi)-\nabla_{s}(J \eta)=\left(\nabla_{\eta} J\right) \xi-\left(\nabla_{\xi} J\right) \eta
$$

hence

$$
\begin{aligned}
\nabla_{s} \nabla_{s} \xi+\nabla_{t} \nabla_{t} \xi & =\nabla_{s}\left(\nabla_{s} \xi+\nabla_{t} \eta\right)+\nabla_{t} \nabla_{s} \eta-\nabla_{s} \nabla_{t} \eta \\
& =\nabla_{s}\left(\left(\nabla_{\eta} J\right) \xi-\left(\nabla_{\xi} J\right) \eta\right)-R(\xi, \eta) \eta
\end{aligned}
$$


and hence

$$
\Delta w=\left|\nabla_{s} \xi\right|^{2}+\left|\nabla_{t} \xi\right|^{2}-\langle R(\xi, \eta) \eta, \xi\rangle+\kappa
$$

The error term $\kappa$ is

$$
\begin{aligned}
\kappa & =\left\langle\xi, \nabla_{s}\left(\left(\nabla_{\eta} J\right) \xi-\left(\nabla_{\xi} J\right) \eta\right)\right\rangle \\
& =\left\langle\xi,\left(\left(\nabla_{\eta} J\right) \nabla_{s} \xi-\left(\nabla_{\xi} J\right) \nabla_{s} \eta\right)\right\rangle+\left\langle\xi,\left(\nabla_{s}\left(\nabla_{\eta} J\right)\right) \xi-\left(\nabla_{s}\left(\nabla_{\xi} J\right) \eta\right)\right\rangle .
\end{aligned}
$$

There exists a constant $c=c(M, J, g)>0$ such that

$$
\left|\nabla_{s}\left(\nabla_{\eta} J\right)\right| \leqslant c\left(|\xi|^{2}+\left|\nabla_{t} \xi\right|\right), \quad\left|\nabla_{s}\left(\nabla_{\xi} J\right)\right| \leqslant c\left(|\xi|^{2}+\left|\nabla_{s} \xi\right|\right) .
$$

Hence there exists a constant $c^{\prime}=c^{\prime}(M, J, g)>0$ such that

$$
\begin{aligned}
\kappa & \geqslant-c^{\prime}|\xi|^{4}-c^{\prime}|\xi|^{2}\left|\nabla_{s} \xi\right|-c^{\prime}|\xi|^{2}\left|\nabla_{t} \xi\right| \\
& \geqslant-\frac{1}{2}\left|\nabla_{s} \xi\right|^{2}-\frac{1}{2}\left|\nabla_{t} \xi\right|^{2}-c^{\prime}\left(1+c^{\prime}\right)|\xi|^{4} .
\end{aligned}
$$

By (B.1), there exists a constant $c^{\prime \prime}=c^{\prime \prime}(M, J, g)>0$ such that

$$
\Delta w \geqslant-c^{\prime \prime}|\xi|^{4} / 4=-c^{\prime \prime} w^{2}
$$

Now the normal derivative of $w$ on $H_{r}\left(z_{0}\right) \cap \mathbb{R}$ is zero:

$$
\begin{aligned}
\partial_{t} w(s, 0) & =\left\langle\xi(s, 0), \nabla_{t} \xi(s, 0)\right\rangle \\
& =\left\langle\xi(s, 0), \nabla_{s} \eta(s, 0)\right\rangle \\
& =\left\langle\xi(s, 0), \nabla_{s}(J(u(s, 0)) \xi(s, 0))\right\rangle \\
& =\left\langle\xi(s, 0), J(u(s, 0)) \nabla_{s} \xi(s, 0)\right\rangle \\
& =0 .
\end{aligned}
$$

The penultimate equality uses the fact that $\nabla_{\xi} J(u)$ is skew-symmetric with respect to $g$. This follows from condition (i) of Lemma D.1. The last equality uses conditions (ii) and (iii) of Lemma D.1. Namely, since $L$ is totally geodesic we have $\nabla_{s} \xi(s, 0) \in T_{u(s, 0)} L$ and hence, by (ii), $J(u(s, 0)) \nabla_{s} \xi(s, 0)$ is orthogonal to $T_{u(s, 0)} L$. It follows that $w$ extends by reflection to a twice continuously differentiable function on the open disc $B_{r}\left(z_{0}\right):=$ $\left\{z \in \mathbb{C}|| z-z_{0} \mid<r\right\}$. The extended function will still be denoted by $w$. It satisfies $w(\bar{z})=w(z)$ and $\Delta w \geqslant-c^{\prime \prime} w^{2}$. Hence the assertion follows from Lemma A. 1 .

Remark B.2. - If $(M, J, g)$ is a Kähler manifold then the error term $\kappa$ in Eq. (B.1) vanishes. If, in addition, the curvature is negative then $w$ is subharmonic and Lemma A.1 is not required.

Next we want to allow for $J$ to depend on both $z=s+i t$ and $u$. Following Gromov [10] we do this by introducing an almost complex structure on the product $\mathbb{H} \times M$. In our application we do not need the vector fields $X$ and $Y$ below.

Let $\left\{J_{z}\right\}_{z \in \mathbb{H}}$ be a smooth family of almost complex structures on $M$ that has compact support in the sense that there exist a constant $R>0$ and an almost complex structure $J_{\infty}$ on $M$ such that $J_{z}=J_{\infty}$ for $|z| \geqslant R$. We assume that $J_{z} \in \mathcal{J}(M, L)$ for every $z \in \mathbb{R}$. 
LEMMA B.3. - Under these hypotheses there exist constants $\delta>0$ and $c>0$ such that the following holds for every $r \in(0, \delta)$, every $z_{0} \in \mathbb{H}$, and every smooth map $u: H_{r}\left(z_{0}\right) \rightarrow M$. If $u$ satisfies the boundary value problem

$$
\partial_{s} u+J_{s, t}(u) \partial_{t} u=0, \quad u(s, 0) \in L,
$$

then

$$
\int_{H_{r}\left(z_{0}\right)}\left|\partial_{s} u\right|^{2}<\delta \Longrightarrow\left|\partial_{s} u\left(z_{0}\right)\right|^{2} \leqslant c\left(1+\frac{1}{r^{2}} \int_{H_{r}\left(z_{0}\right)}\left|\partial_{s} u\right|^{2}\right) .
$$

Proof. - Denote

$$
\widetilde{M}:=\mathbb{H} \times M, \quad \widetilde{L}:=\mathbb{R} \times L,
$$

and consider the almost complex structure $\widetilde{J}$ on $\widetilde{M}$ given by

$$
\widetilde{J}=\left(\begin{array}{ccc}
0 & -1 & 0 \\
1 & 0 & 0 \\
0 & 0 & J
\end{array}\right)
$$

Then $\widetilde{L}$ is a totally real submanifold of $(\widetilde{M}, \widetilde{J})$ and $u$ satisfies (B.2) if and only if the function $\tilde{u}: H_{r}\left(z_{0}\right) \rightarrow \widetilde{M}$, defined by

$$
\tilde{u}(s, t):=(s, t, u(s, t))
$$

is a $\widetilde{J}$-holomorphic curve in $\widetilde{M}$ with boundary values in $\widetilde{L}$.

Lemma B.1 does not immediately apply in the present situation as $\widetilde{M}$ is not compact. However, we may argue as follows. It suffices to prove the lemma under the additional hypothesis that $\left|z_{0}\right| \leqslant R+1$. Namely, if $\left|z_{0}\right|>R+1$ replace $z_{0}$ by $z_{1}$, where $\left|z_{1}\right|=R+1$ and $u$ by $u_{1}(z)=u\left(z+z_{0}-z_{1}\right)$. Now $\widetilde{M} \cap\left(B_{R+1}(0) \times M\right)$ may be identified with an open subset of a closed manifold and $\widetilde{L} \cap\left(B_{R+1}(0) \times M\right)$ and $\widetilde{J}$ may be extended.

Now we can apply Lemma B.1, i.e. there exist constants $\delta>0$ and $c>0$ such that the conclusion of Lemma B.1 holds with $M, L$, and $J$ replaced by $\widetilde{M}, \widetilde{L}$, and $\widetilde{J}$, and with the product metric on $\widetilde{M}$. Note that

$$
\left|\partial_{s} \tilde{u}(s, t)\right|^{2}=1+\left|\partial_{s} u(s, t)\right|^{2} .
$$

Assume

$$
r<\sqrt{\frac{\delta}{2 \pi}}, \quad \int_{H_{r}\left(z_{0}\right)}\left|\partial_{s} u\right|^{2}<\frac{\delta}{2} .
$$

Then

$$
\int_{H_{r}\left(z_{0}\right)}\left|\partial_{s} \tilde{u}\right|^{2} \leqslant \pi r^{2}+\int_{H_{r}\left(z_{0}\right)}\left|\partial_{s} u\right|^{2}<\delta
$$


Hence, by Lemma B.1,

$$
\left|\partial_{s} u\left(z_{0}\right)\right|^{2} \leqslant\left|\partial_{s} \tilde{u}\left(z_{0}\right)\right|^{2} \leqslant \frac{c}{r^{2}} \int_{H_{r}\left(z_{0}\right)}\left|\partial_{s} \tilde{u}\right|^{2} \leqslant \frac{c}{\pi}+\frac{c}{r^{2}} \int_{H_{r}\left(z_{0}\right)}\left|\partial_{s} u\right|^{2} .
$$

This proves the lemma.

\section{Appendix C. Elliptic bootstrapping}

In this section we shall prove that a uniform bound on the first derivatives of $u$ gives rise to uniform bounds on the higher derivatives whenever $u$ is a solution of (B.2). The proof uses the $L^{2}$-estimate for the Laplace operator. Throughout $\Omega$ denotes a bounded open subset of $\mathbb{H}$. Note that $\Omega \cap \mathbb{R}$ may be nonempty. Let

$$
J_{0}:=\left(\begin{array}{cc}
0 & -\mathbb{1} \\
\mathbb{1} & 0
\end{array}\right) \in \mathbb{R}^{2 n \times 2 n}
$$

denote the standard complex structure.

We consider the Cauchy-Riemann operator

$$
\bar{\partial} \xi=\partial_{s} \xi+J_{0} \partial_{t} \xi
$$

for smooth functions $\xi: \Omega \rightarrow \mathbb{R}^{2 n}$ that satisfy the Lagrangian boundary condition

$$
\xi(s, 0) \in \mathbb{R}^{n} \times\{0\}
$$

for all $s \in \mathbb{R}$ such that $(s, 0) \in \Omega$.

LEMMA C.1. - Let $\Omega, \Omega^{\prime}$ be bounded open subsets of $\mathbb{H}$ such that $\bar{\Omega} \subset \Omega^{\prime}$. Then, for every integer $k \geqslant 0$, there exists a constant $c=c\left(k, \Omega, \Omega^{\prime}\right)>0$ such that

$$
\|\xi\|_{W^{k+1,2}(\Omega)} \leqslant c\left(\|\bar{\partial} \xi\|_{W^{k, 2}\left(\Omega^{\prime}\right)}+\|\xi\|_{W^{k, 2}\left(\Omega^{\prime}\right)}\right)
$$

for every smooth function $\xi: \Omega^{\prime} \rightarrow \mathbb{R}^{2 n}$ that satisfies (C.1).

Proof. - Assume first that $\xi$ has compact support in $\Omega^{\prime}$. Then it follows from the boundary condition and integration by parts that

$$
\begin{aligned}
\int_{\Omega^{\prime}}|\bar{\partial} \xi|^{2} & =\int_{\Omega^{\prime}}\left(\left|\partial_{s} \xi\right|^{2}+\left|\partial_{t} \xi\right|^{2}+2\left\langle\partial_{s} \xi, J_{0} \partial_{t} \xi\right\rangle\right) \\
& =\int_{\Omega^{\prime}}\left(\left|\partial_{s} \xi\right|^{2}+\left|\partial_{t} \xi\right|^{2}+\left\langle\partial_{s} \xi, J_{0} \partial_{t} \xi\right\rangle-\left\langle\xi, J_{0} \partial_{s} \partial_{t} \xi\right\rangle\right) \\
& =\int_{\Omega^{\prime}}\left(\left|\partial_{s} \xi\right|^{2}+\left|\partial_{t} \xi\right|^{2}+\left\langle\partial_{s} \xi, J_{0} \partial_{t} \xi\right\rangle+\left\langle\partial_{t} \xi, J_{0} \partial_{s} \xi\right\rangle\right) \\
& =\int_{\Omega^{\prime}}\left(\left|\partial_{s} \xi\right|^{2}+\left|\partial_{t} \xi\right|^{2}\right) .
\end{aligned}
$$


Hence, by Poincaré's inequality, there exists a constant $c_{1}=c_{1}\left(\Omega^{\prime}\right)>0$ such that

$$
\|\xi\|_{W^{1,2}\left(\Omega^{\prime}\right)} \leqslant c_{2}\left\|\partial_{s} \xi+J_{0} \partial_{t} \xi\right\|_{L^{2}\left(\Omega^{\prime}\right)}
$$

for every smooth function $\xi: \Omega^{\prime} \rightarrow \mathbb{R}^{2 n}$ with compact support that satisfies (C.1). If $\xi$ does not have compact support choose a smooth cutoff function $\beta: \Omega^{\prime} \rightarrow \mathbb{R}$ with compact support such that $\beta=1$ on $\Omega$, and apply the previous inequality to $\beta \xi$ to obtain (C.2) for $k=0$. Assume, by induction, that (C.2) has been established for some integer $k \geqslant 0$. Then (C.2) with $k$ replaced by $k+1$ follows by applying (C.2) to the function $\partial_{s} \xi$.

Next we shall consider the Laplace operator

$$
\Delta=\frac{\partial^{2}}{\partial s^{2}}+\frac{\partial^{2}}{\partial t^{2}}
$$

for smooth functions $u: \Omega \rightarrow \mathbb{R}$ that satisfy either the Dirichlet boundary conditions

$$
u(s, 0)=0
$$

for all $s \in \mathbb{R}$ such that $(s, 0) \in \Omega$ or the Neumann boundary condition

$$
\partial_{t} u(s, 0)=0
$$

for all $s \in \mathbb{R}$ such that $(s, 0) \in \Omega$.

LemmA C.2. - Let $\Omega, \Omega^{\prime \prime}$ be bounded open subsets of $\mathbb{H}$ such that $\bar{\Omega} \subset \Omega^{\prime \prime}$. Then, for every integer $k \geqslant 0$, there exists a constant $c=c\left(k, \Omega, \Omega^{\prime \prime}\right)>0$ such that

$$
\|u\|_{W^{k+2,2(\Omega)}} \leqslant c\left(\|\Delta u\|_{W^{k, 2}\left(\Omega^{\prime \prime}\right)}+\|u\|_{W^{k+1,2\left(\Omega^{\prime \prime}\right)}}\right)
$$

for every smooth function $u: \Omega^{\prime} \rightarrow \mathbb{R}$ that satisfies either (C.3) or (C.4).

Proof. - Choose an open set $\Omega^{\prime} \subset \mathbb{H}$ such that $\bar{\Omega} \subset \Omega^{\prime}$ and $\bar{\Omega}^{\prime} \subset \Omega^{\prime \prime}$. Denote

$$
\partial:=\partial_{s}-J_{0} \partial_{t} .
$$

By Lemma C.1, there exist constants $c^{\prime}$ and $c^{\prime \prime}$ such that

$$
\begin{gathered}
\|\xi\|_{W^{k+2,2}(\Omega)} \leqslant c^{\prime}\left(\|\bar{\partial} \xi\|_{W^{k+1,2}\left(\Omega^{\prime}\right)}+\|\xi\|_{W^{k+1,2}\left(\Omega^{\prime}\right)}\right), \\
\|\eta\|_{W^{k+1,2}\left(\Omega^{\prime}\right)} \leqslant c^{\prime \prime}\left(\|\partial \eta\|_{W^{k, 2}\left(\Omega^{\prime \prime}\right)}+\|\eta\|_{W^{k, 2}\left(\Omega^{\prime \prime}\right)}\right)
\end{gathered}
$$

for all smooth functions $\xi: \Omega^{\prime} \rightarrow \mathbb{R}^{2}$ and $\eta: \Omega^{\prime \prime} \rightarrow \mathbb{R}^{2}$ that satisfy the boundary condition (C.1). Now suppose that $u, v: \Omega^{\prime \prime} \rightarrow \mathbb{R}$ are smooth functions such that $v$ satisfies the Dirichlet boundary condition and $u$ satisfies the Neumann boundary condition, i.e.

$$
\partial_{t} u(s, 0)=v(s, 0)=0
$$


for $s \in \mathbb{R}$ such that $(s, 0) \in \Omega^{\prime \prime}$. Then

$$
\xi:=(u, v): \Omega^{\prime \prime} \rightarrow \mathbb{R}^{2}
$$

satisfies the Lagrangian boundary condition (C.1) and so does the function $\eta:=\bar{\partial} \xi$. Since $\Delta=\partial \bar{\partial}$ it follows that

$$
\begin{aligned}
\|\xi\|_{W^{k+2,2}(\Omega)} & \leqslant c^{\prime}\left(\|\bar{\partial} \xi\|_{W^{k+1,2}\left(\Omega^{\prime}\right)}+\|\xi\|_{W^{k+1,2}\left(\Omega^{\prime}\right)}\right) \\
& \leqslant c^{\prime}\left(c^{\prime \prime}\|\Delta \xi\|_{W^{k, 2}\left(\Omega^{\prime \prime}\right)}+\left(1+c^{\prime \prime}\right)\|\xi\|_{W^{k+1,2}\left(\Omega^{\prime \prime}\right)}\right) .
\end{aligned}
$$

This proves the lemma.

LEMMA C.3. - Let $\Omega, \Omega^{\prime}$ be bounded open subsets of $\mathbb{H}$ such that $\bar{\Omega} \subset \Omega^{\prime}$. Let $M, L$, $\left\{J_{z}\right\}_{z \in \Omega^{\prime}}$, be as in the hypotheses of Lemma B.3. Then, for every integer $k \geqslant 0$ and every constant $c_{1}>0$, there exists a constant $c_{k}=c_{k}\left(c_{1}, \Omega, \Omega^{\prime}\right)>0$ such that the following holds for every smooth map $u: \Omega^{\prime} \rightarrow$ M. If u satisfies the boundary value problem (B.2) then

$$
\sup _{\Omega^{\prime}}\left|\partial_{s} u\right| \leqslant c_{1} \Longrightarrow\|u\|_{C^{k}(\Omega)} \leqslant c_{k} .
$$

Proof. - Let $2 n=\operatorname{dim} M$ and $n=\operatorname{dim} L$. Cover $M$ by finitely many coordinate charts that identify $L$ with $\mathbb{R}^{n} \times\{0\} \subset \mathbb{R}^{n}$ and identify $J$ with the standard complex structure $J_{0}: R^{2 n} \rightarrow \mathbb{R}^{2 n}$ on $\mathbb{R}^{n} \times\{0\} \cong L$. (Do this by choosing any coordinates $x_{1}, \ldots, x_{n}$ on $L$ and using exponential normal coordinates $y_{1}, \ldots, y_{n}$ with $\partial / \partial y_{i}=J \partial / \partial x_{i}$.) Choose $\delta>0$ such that

$$
z_{0} \in \Omega \Longrightarrow H_{\delta}\left(z_{0}\right) \subset \Omega^{\prime}
$$

and that, for every $p \in M$, there exists a coordinate chart as above that contains the closed ball of radius $\delta c_{1}$ about $p$.

Now let $u: \Omega^{\prime} \rightarrow M$ be a solution of (B.2) such that

$$
\sup _{\Omega^{\prime}}\left|\partial_{s} u\right| \leqslant c_{1}
$$

and fix a point $z_{0} \in \Omega$. Then $d\left(u\left(z_{0}\right), u(z)\right) \leqslant c_{1} \delta$ for every $z \in H_{\delta}\left(z_{0}\right)$. Hence there is a coordinate chart as above that contains $u\left(H_{\delta}\left(z_{0}\right)\right)$. Write $u: H_{\delta}\left(z_{0}\right) \rightarrow U \subset \mathbb{R}^{2 n}$ for the map $u$ in this coordinate chart. Then

$$
\begin{gathered}
\partial_{s} u+J(s, t, u) \partial_{t} u=0, \\
u(s, 0) \in \mathbb{R}^{n} \times\{0\} .
\end{gathered}
$$

We prove by induction that, for every $k$, there exists a constant $c_{k}$, independent of $u$, such that

$$
\|u\|_{W^{k, 2}\left(H_{\delta / k}\left(z_{0}\right)\right)} \leqslant c_{k} .
$$

Abbreviate $J=J(s, t, u(s, t))$ and think of this as a functions of $s$ and $t$. Then, by (C.5),

$$
\left(\partial_{s}-J \partial_{t}\right)\left(\partial_{s} u+J \partial_{t} u\right)=0
$$


and hence

$$
\Delta u=\left(\partial_{s} J\right) \partial_{t} u-\left(\partial_{t} J\right) \partial_{s} u .
$$

Write $u=\left(u_{1}, u_{2}\right)$ where $u_{i}: \mathbb{H}_{\delta}\left(z_{0}\right) \rightarrow \mathbb{R}^{n}$ for $i=1,2$. Then, by (C.6), $u_{2}(s, 0)=0$ and hence

$$
\partial_{t} u_{1}(s, 0)=-\partial_{s} u_{2}(s, 0)=0,
$$

i.e. $u_{1}$ satisfies the Neumann boundary condition and $u_{2}$ satisfies the Dirichlet boundary condition. Hence it follows from Lemma C. 2 that for every integer $k \geqslant 1$ there exists a constant $c_{k+1}^{\prime}$ such that

$$
\|u\|_{W^{k+1,2}\left(H_{\delta /(k+1)}\left(z_{0}\right)\right)} \leqslant c_{k+1}^{\prime}\left(\|\Delta u\|_{W^{k-1,2}\left(H_{\delta / k}\left(z_{0}\right)\right)}+\|u\|_{W^{k, 2}\left(H_{\delta / k}\left(z_{0}\right)\right)}\right) .
$$

By assumption there exists a constant $c_{1}^{\prime}$, depending only on $c_{1}$ and the choice of the coordinate charts, such that

$$
\|u\|_{C^{1}\left(H_{\delta}\left(z_{0}\right)\right)} \leqslant c_{1}^{\prime} .
$$

Hence it follows from (C.9) with $k=1$ and (C.8) that (C.7) holds with $k=2$. Now the formula (C.8) shows that the $W^{2,2}$-bound on $u$ together with the $C^{1}$-bound implies a $W^{1,2}$-bound on $\Delta u$ in the domain $H_{\delta / 2}\left(z_{0}\right)$. Hence it follows from (C.9) with $k=2$ that (C.7) holds with $k=3$. Now suppose, by induction, that (C.7) has been established for $k \geqslant 3$. Then, by (C.8), $\Delta u$ satisfies a $W^{k-1,2}$-bound in the domain $H_{\delta / k}\left(z_{0}\right)$ and hence, by (C.9), $u$ satisfies a $W^{k+1,2}$-bound in $H_{\delta /(k+1)}\left(z_{0}\right)$. This proves (C.7). With (C.7) established, the assertion of the lemma follows from the Sobolev inequality

$$
\|u\|_{C^{0}\left(H_{r}\left(z_{0}\right)\right)} \leqslant c_{0}(r)\|u\|_{W^{2,2}\left(H_{r}\left(z_{0}\right)\right)}
$$

for some constant $c_{0}(r)$ and every smooth function $u: H_{r}\left(z_{0}\right) \rightarrow \mathbb{R}^{2 n}$.

\section{Appendix D. A convenient metric}

The following lemma appeared in the Diploma thesis of Urs Frauenfelder [9]. We give a proof for the convenience of the reader.

Lemma D.1 (Urs Frauenfelder). - Let $(M, J)$ be an almost complex manifold and $L \subset M$ be a totally real submanifold with $2 \operatorname{dim} L=\operatorname{dim} M$. Then there exists $a$ Riemannian metric $g$ on $M$ such that

(i) $g(J(p) v, J(p) w)=g(v, w)$ for $p \in M$ and $v, w \in T_{p} M$,

(ii) $J(p) T_{p} L$ is the orthogonal complement of $T_{p} L$ for every $p \in L$,

(iii) $L$ is totally geodesic with respect to $g$.

Proof. - Choose coordinates $x_{1}, \ldots, x_{n}$ on $L$ and extend these to coordinates $x_{1}, \ldots$, $x_{n}, y_{1}, \ldots, y_{n}$ on $M$ such that

$$
J \frac{\partial}{\partial x_{i}}=\frac{\partial}{\partial y_{i}}, \quad i=1, \ldots, n,
$$


on $L$. Write a metric in these coordinates in the form

$$
g(x, y)=\left(\begin{array}{cc}
a(x, y) & b(x, y)^{T} \\
b(x, y) & c(x, y)
\end{array}\right)
$$

where $a(x, y)=a(x, y)^{T}, b(x, y)$, and $c(x, y)=c(x, y)^{T}$ are real $n \times n$-matrices. Such a metric satisfies (i), (ii), and (iii) if and only if

$$
a(x, 0)=c(x, 0), \quad b(x, 0)=0, \quad \partial_{n+i} a(x, 0)=0,
$$

for $i=1, \ldots, n$. The set of metrics that satisfy (D.1) is invariant under convex combinations and under multiplication by cutoff functions $\beta=\beta(x, y)$ that satisfy

$$
\partial_{n+i} \beta(x, 0)=0 .
$$

This condition on the cutoff function is intrinsic. It asserts that

$$
q \in L, v \in T_{q} L \Longrightarrow d \beta(q) J(q) v=0 .
$$

Hence the result follows by choosing local metrics that satisfy (D.1) and patching with a partition of unity consisting of finitely many cutoff functions that satisfy (D.2).

\section{Appendix E. Applications to contact geometry}

Let $M$ be a $2 n+1$-dimensional oriented manifold and $\alpha \in \Omega^{1}(M)$ be a contact form, i.e.

$$
\alpha \wedge(d \alpha)^{n}>0
$$

The Reeb vector field $Y$ of $\alpha$ is defined by

$$
\iota(Y) \alpha=1, \quad \iota(Y) d \alpha=0 .
$$

We denote by $\varphi: \mathbb{R} \times M \rightarrow M$ the Reeb flow and write $\varphi^{\tau}(p):=\varphi(\tau, p)$ whenever convenient. Let $L \subset M$ be a closed Legendrian submanifold, i.e. $\left.\alpha\right|_{T L}=0$ and $L$ has dimension $n$. A characteristic chord is a pair $(T, \gamma)$, where $\gamma:[0,1] \rightarrow M$ is a smooth curve and $T>0$ is a real number such that

$$
\dot{\gamma}(t)=T Y(\gamma(t)), \quad \gamma(0) \in L, \quad \gamma(1) \in L .
$$

In particular, $\gamma(t)=\varphi(t T, \gamma(0))$ for $0 \leqslant t \leqslant 1$. We allow the possibility that the image of the characteristic chord lies on a periodic orbit of the Reeb flow and hence $\gamma$ may not be injective. Call the characteristic chord nondegenerate if $\varphi: \mathbb{R} \times L \rightarrow M$ is transverse to $L$ at the point $(T, \gamma(0))$.

Now suppose that $J: T M \rightarrow T M$ is an endomorphism such that

$$
\alpha \circ J=0, \quad J Y=0, \quad J^{2}=\alpha Y-\mathbb{1},
$$


and the formula

$$
\langle v, w\rangle:=d \alpha(v, J w)+\alpha(v) \alpha(w)
$$

defines a Riemannian metric on $M$. In particular, $J$ maps the kernel of $\alpha$ to itself and its restriction to the kernel of $\alpha$ is an almost complex structure that is compatible with the symplectic form $d \alpha$. As in Section 1 let us denote the half strip by $S=[0, \infty) \times[0,1]$. We consider the partial differential equation

$$
\begin{gathered}
\partial_{s} u-\alpha\left(\partial_{s} u\right) Y(u)+J(u)\left(\partial_{t} u-\alpha\left(\partial_{t} u\right) Y(u)\right)=0, \\
\partial_{s} \alpha\left(\partial_{s} u\right)+\partial_{t} \alpha\left(\partial_{t} u\right)=0,
\end{gathered}
$$

for smooth functions $u: S \rightarrow M$ that satisfy the boundary condition

$$
u(s, 0) \in L, \quad u(s, 1) \in L,
$$

for $s \geqslant 0$.

Remark E.1. - The solutions of (E.2) and (E.3) correspond to pseudoholomorphic curves in the symplectization as follows. Consider the symplectic manifold $(\widetilde{M}, \widetilde{\omega})$ defined by

$$
\widetilde{M}:=\mathbb{R} \times M, \quad \widetilde{\omega}:=d\left(\mathrm{e}^{\theta} \alpha\right),
$$

where $\theta$ denotes the $\mathbb{R}$-coordinate. Then $\widetilde{L}:=\mathbb{R} \times L$ is a Lagrangian submanifold of $\widetilde{M}$ and the automorphism $\widetilde{J}: T \widetilde{M} \rightarrow T \widetilde{M}$ defined by

$$
\widetilde{J}(\hat{\theta}, v):=(-\alpha(v), J v+\hat{\theta} Y)
$$

is an almost complex structure on $\widetilde{M}$ that is compatible with $\widetilde{\omega}$. The corresponding Riemannian metric on $\widetilde{M}=\mathbb{R} \times M$ is $\mathrm{e}^{\theta}$ times the product metric, where the metric on $M$ is given by (E.1). Now $u: S \rightarrow M$ satisfies (E.2) and (E.3) if and only if there exists a smooth function $\theta: S \rightarrow \mathbb{R}$ such that the function $\widetilde{u}: S \rightarrow \widetilde{M}$, defined by

$$
\widetilde{u}(s, t):=(\theta(s, t), u(s, t))
$$

is a $\widetilde{J}$-holomorphic curve in $\widetilde{M}$ with boundary values in $\widetilde{L}$. The function $\theta$ is determined by the equations

$$
\partial_{s} \theta=\alpha\left(\partial_{t} u\right), \quad \partial_{t} \theta=-\alpha\left(\partial_{s} u\right)
$$

up to an additive constant.

Lemma E.2. - Assume $M$ is compact and that all characteristic chords $(T, \gamma)$ are nondegenerate. Let $u: S \rightarrow M$ be a solution of (E.2) and (E.3) such that

$$
\lim _{s \rightarrow \infty} \theta(s, t)=\infty, \quad 0<E_{0}(u)<\infty,
$$


where $\theta: S \rightarrow \mathbb{R}$ satisfies (E.4) and

$$
E_{0}(u)=\int u^{*} d \alpha=\int_{0}^{1} \int_{0}^{\infty}\left|\partial_{s} u-\alpha\left(\partial_{s} u\right) Y(u)\right|^{2} d s d t
$$

Then there exists a characteristic chord $(T, \gamma)$ such that

$$
\lim _{s \rightarrow \infty} u(s, t)=\gamma(t), \quad \lim _{s \rightarrow \infty} \alpha\left(\partial_{t} u(s, t)\right)=T
$$

The limits are uniform in $t$.

Proof. - By [6, Theorem 5.9] (the numbering refers to the first draft), every sequence $s_{v} \rightarrow \infty$ has a subsequence $s_{v_{i}}$ such that $\left(\alpha\left(\partial_{t} u\left(s_{v_{i}}, \cdot\right)\right), u\left(s_{v_{i}}, \cdot\right)\right)$ converges uniformly to a characteristic chord $(T, \gamma)$. By nondegeneracy, characteristic chords are isolated. Hence the limit is independent of the sequence $s_{v}$ and of the subsequence $s_{v_{i}}$.

Fix a nondegenerate characteristic chord $(T, \gamma)$ and a function $u: S \rightarrow M$ that satisfies (E.5). It is convenient to introduce the following coordinates in a sufficiently small neighbourhood $U$ of the image of $\gamma$. Choose a local submanifold $\bar{M} \subset M$ of dimension $2 n$ such that

$$
\bar{p}:=\gamma(0) \in \bar{M}, \quad T_{\bar{p}} \bar{M}=\operatorname{ker} \alpha
$$

and the Reeb flow defines an open immersion

$$
\varphi:(-\varepsilon, T+\varepsilon) \times \bar{M} \rightarrow U
$$

onto an open neighbourhood $U$ of $\gamma([0,1])$. The manifold $\bar{M}$ carries an exact symplectic form

$$
\bar{\omega}:=d \bar{\alpha}, \quad \bar{\alpha}:=\left.\alpha\right|_{\bar{M}} .
$$

If $\bar{M}$ is chosen as a sufficiently small slice that is transverse to $\gamma$ then there are two unique Lagrangian submanifolds $\bar{L}_{0}, \bar{L}_{1} \subset \bar{M}$ and two smooth functions

$$
\tau_{0}: \bar{L}_{0} \rightarrow \mathbb{R}, \quad \tau_{1}: \bar{L}_{1} \rightarrow \mathbb{R}
$$

such that $\varphi^{\tau_{0}(\bar{q})}(\bar{q}) \in U \cap L$ for $\bar{q} \in \bar{L}_{0}, \varphi^{\tau_{1}(\bar{q})}(\bar{q}) \in U \cap L$ for $\bar{q} \in \bar{L}_{1}$, and

$$
\tau_{0}(\bar{p})=0, \quad \tau_{1}(\bar{p})=T
$$

Since $\gamma$ is nondegenerate, these Lagrangian submanifolds intersect transversally at $\bar{p} \in \bar{L}_{0} \cap \bar{L}_{1}$ and they do not intersect in any other point. For $\tau \in(-\varepsilon, T+\varepsilon)$ define the almost complex structure $\bar{J}_{\tau}=\bar{J}(\tau, \cdot)$ on $\bar{M}$ by

$$
d \varphi^{\tau}(\bar{q})\left(\bar{J}_{\tau}(\bar{q}) \bar{v}-\alpha\left(\bar{J}_{\tau}(\bar{q}) \bar{v}\right) Y(\bar{q})\right)=J\left(\varphi^{\tau}(\bar{q})\right) d \varphi^{\tau}(\bar{q}) \bar{v}
$$


for $\bar{v} \in T_{\bar{q}} \bar{M}$. Then $\bar{J}_{\tau}$ is compatible with $\bar{\omega}$ for every $\tau$. Let us now assume that $u(s, t) \in U$ for every $s+i t \in S$ and define $\bar{u}: S \rightarrow \bar{M}$ and $\tau: S \rightarrow \mathbb{R}$ by the condition that $\bar{u}(s, t) \rightarrow \bar{p}$ and $\tau(s, t) \rightarrow t T$ as $s$ tends to $\infty$ and

$$
\varphi(\tau(s, t), \bar{u}(s, t))=u(s, t)
$$

for $s \geqslant 0$ and $0 \leqslant t \leqslant 1$.

LEMMA E.3. - If u satisfies (E.2) and (E.3) then

$$
\partial_{s} \bar{u}+\bar{J}(\tau, \bar{u}) \partial_{t} \bar{u}=0, \quad \Delta \tau+\partial_{s} \bar{\alpha}\left(\partial_{s} \bar{u}\right)+\partial_{t} \bar{\alpha}\left(\partial_{t} \bar{u}\right)=0,
$$

where $\Delta:=\partial_{s}{ }^{2}+\partial_{t}{ }^{2}$, and, for every $s \geqslant 0$,

$$
\bar{u}(s, t) \in \bar{L}_{t}, \quad \tau(s, t)=\tau_{t}(\bar{u}(s, t)), \quad t=0,1 .
$$

Proof. - Differentiate (E.7) to obtain

$$
\partial_{s} u=d \varphi^{\tau}(\bar{u}) \partial_{s} \bar{u}+\left(\partial_{s} \tau\right) Y(u),
$$

hence

$$
\alpha\left(\partial_{s} u\right)=\alpha\left(\partial_{s} \bar{u}\right)+\partial_{s} \tau
$$

and hence

$$
\partial_{s} u-\alpha\left(\partial_{s} u\right) Y(u)=d \varphi^{\tau}(\bar{u})\left(\partial_{s} \bar{u}-\alpha\left(\partial_{s} \bar{u}\right) Y(\bar{u})\right) .
$$

Similar identities hold with $s$ replaced by $t$. Hence, by (E.6),

$$
\begin{aligned}
0 & =\partial_{s} u-\alpha\left(\partial_{s} u\right) Y(u)+J(u)\left(\partial_{t} u-\alpha\left(\partial_{t} u\right) Y(u)\right) \\
& =d \varphi^{\tau}(\bar{u})\left(\partial_{s} \bar{u}-\alpha\left(\partial_{s} \bar{u}\right) Y(\bar{u})\right)+J\left(\varphi^{\tau}(\bar{u})\right) d \varphi^{\tau}(\bar{u}) \partial_{t} \bar{u} \\
& =d \varphi^{\tau}(\bar{u})\left(\partial_{s} \bar{u}-\alpha\left(\partial_{s} \bar{u}\right) Y(\bar{u})+\bar{J}_{\tau}(\bar{u}) \partial_{t} \bar{u}-\alpha\left(\bar{J}_{\tau}(\bar{u}) \partial_{t} \bar{u}\right) Y(\bar{u})\right) \\
& =d \varphi^{\tau}(\bar{u})\left(\partial_{s} \bar{u}+\bar{J}_{\tau}(\bar{u}) \partial_{t} \bar{u}\right)-\alpha\left(\partial_{s} \bar{u}+\bar{J}_{\tau}(\bar{u}) \partial_{t} \bar{u}\right) Y\left(\varphi^{\tau}(\bar{u})\right) .
\end{aligned}
$$

Since $Y\left(\varphi^{\tau}(\bar{p})\right)$ is not contained in the image of $T_{\bar{p}} \bar{M}$ under the differential $d \varphi^{\tau}(\bar{p})$, it follows that $\partial_{s} \bar{u}+\bar{J}_{\tau}(\bar{u}) \partial_{t} \bar{u}=0$. This proves the first equation in (E.8) and the second equation follows from (E.10). The boundary condition (E.9) follows directly from the definitions. This proves the lemma.

The first equation in (E.8) differs from (CR) in that $\tau$ depends on $s$. In the special case where $J$ is invariant under the Reeb flow, $\bar{J}_{\tau}$ is independent of $\tau$ and hence the asymptotic behaviour of $\bar{u}$ follows directly from Theorem $\mathrm{B}$. The asymptotic behaviour of $\tau$ can then be deduced from the second equation in (E.8). In this special case the results of Abbas [1] follow from ours. We believe that in general the results of [1] can be derived (and extended to higher dimensions) by allowing $J$ to depend on $s$ and using elliptic bootstrapping arguments for the two equations in (E.8).

In the case of a Legendrian knot the contact form and the Reeb vector field are given by

$$
\alpha=d z-y d x, \quad Y=\frac{\partial}{\partial z} .
$$


Consider the endomorphism $J: T \mathbb{R}^{3} \rightarrow T \mathbb{R}^{3}$ defined by

$$
J \frac{\partial}{\partial x}=\frac{\partial}{\partial y}, \quad J \frac{\partial}{\partial y}=-\frac{\partial}{\partial x}-y \frac{\partial}{\partial z}, \quad J \frac{\partial}{\partial z}=0 .
$$

Then Eqs. (E.2) have the form

$$
\partial_{s} u-\partial_{t} v=0, \quad \partial_{s} v+\partial_{t} u=0, \quad \Delta w=0
$$

Here we read $(u, v, w)$ for $u$ in (E.2), $(u, v)$ for $\bar{u}$ in (E.8), and $w$ for $\tau$ in (E.8). The slice $\bar{M}$ is the $(x, y)$-plane and so $\tau=z$. The boundary condition (E.3) has the form

$$
(u(s, 0), v(s, 0), w(s, 0)) \in L, \quad(u(s, 1), v(s, 1), w(s, 1)) \in L,
$$

where $L \subset \mathbb{R}^{3}$ is a Legendrian knot. Every Legendrian knot $L \subset \mathbb{R}^{3}$ projects to an immersed curve $\bar{L}$ in the $(x, y)$-plane and the characteristic chords correspond to the self-intersections of $\bar{L}$. They are nondegenerate if and only if $\bar{L}$ has transverse selfintersections. In this case we can apply Theorem $\mathrm{C}$ to derive the asymptotic behaviour for the map $(u, v)$ and use the Dirichlet boundary value problem to deduce the asymptotic behaviour for $w$.

\section{REFERENCES}

[1] Abbas C., Finite energy surfaces and the chord problem, Duke Math. J. 96 (1999) 241-316.

[2] Agmon S., Nirenberg L., Lower bounds and uniqueness theorems for solutions of differential equations in Hilbert space, Comm. Pure Appl. Math. 20 (1967) 207-229.

[3] Chekanov Y., Differential algebras of Legendrian links, Preprint, Fields Institute, Toronto, 1997.

[4] Eliashberg Y., in preparation.

[5] Eliashberg Y., Givental A., Hofer H., Contact homology, in preparation.

[6] Eliashberg Y., Hofer H., Salamon D., Lagrangian intersection in contact geometry, Geometric and Functional Analysis 5 (1995) 244-269; See also the First Draft on http: //www.math.ethz.ch/ ${ }^{\text {ssalamon. }}$

[7] Floer A., The unregularized gradient flow of the symplectic action, Comm. Pure Appl. Math. 41 (1988) 775-813.

[8] Floer A., Morse theory for Lagrangian intersections, J. Differential Geom. 28 (1988) 513547.

[9] Frauenfelder U., Gromov convergence of pseudoholomorphic discs, Diploma thesis, ETH Zürich, 2000.

[10] Gromov M., Pseudo holomorphic curves in symplectic manifolds, Invent. Math. 82 (1985) 307-347.

[11] Heinz E., Über die Eindeutigkeit beim Cauchyschen Anfangswertproblem einer elliptischen Differentialgleichung zweiter Ordnung, Nachr. Akad. Wiss. Göttingen Math.-Phys. Kl. IIa (1955) 1-12.

[12] Hofer H., Pseudoholomorphic curves in symplectizations with applications to the Weinstein conjecture in dimension three, Invent. Math. 114 (1993) 515-563.

[13] Hofer H., Wysocki K., Zehnder E., Properties of pseudoholomorphic curves in symplectizations I: Asymptotics, Ann. Inst. Henri Poincaré, Analyse Nonlinéaire 13 (1996) 337-379. 
[14] McDuff D., Salamon D., J-holomorphic Curves and Quantum Cohomology, AMS University Lecture Series, Vol. 6, 1994.

[15] Oh Y.-G., Removal of boundary singularities of pseudo-holomorphic curves with Lagrangian boundary conditions, Comm. Pure Appl. Math. 45 (1992) 121-139.

[16] Salamon D., Morse theory, the Conley index and Floer homology, Bulletin L.M.S. 22 (1990) 113-140.

[17] Salamon D., Lectures on Floer Homology, Lecture Notes for the IAS/PCMI Graduate Summer School on Symplectic Geometry and Topology, December 1997, in: Eliashberg Y., Traynor L. (Eds.), Symplectic Geometry and Topology, IAS/Park City Mathematics Series, Vol. 7, 1999, pp. 143-230.

[18] De Silva V., Products in the symplectic Floer homology of Lagrangian intersections, PhD Thesis, Oxford, 1998.

[19] Uhlenbeck K., Removable singularities in Yang-Mills fields, Comm. Math. Phys. 83 (1982) 11-29. 